\title{
Dynamic and Oscillatory Motions of Cable-Driven Parallel Robots based on a Non-Linear Cable Tension Model
}

\author{
Sana Baklouti \\ Université Bretagne-Loire, \\ INSA-LGCGM-EA 3913, \\ 20, avenue des Buttes de Cöesmes, \\ 35043 RENNES Cedex \\ Email: sana.baklouti@insa-rennes.fr \\ Eric Courteille \\ Université Bretagne-Loire, \\ INSA-LGCGM-EA 3913, \\ 20, avenue des Buttes de Cöesmes, \\ 35043 RENNES Cedex, France \\ Email: eric.courteille@insa-rennes.fr \\ Stéphane Caro \\ CNRS, Laboratoire des Sciences du Numrique de Nantes \\ UMR CNRS n6004, \\ 1 , rue de la Noë, \\ 44321 Nantes Cedex 03, France \\ Email: stephane.caro@Is2n.fr \\ Mohamed Dkhil \\ Université Bretagne-Loire, \\ INSA-LGCGM-EA 3913, \\ 20, avenue des Buttes de Cöesmes, \\ 35043 RENNES Cedex \\ Email: mohamed.dkhil@insa-rennes.fr
}

\begin{abstract}
In this paper, dynamic modeling of cable-driven parallel robots (CDPRs) is addressed where each cable length is subjected to variations during operation. It is focusing on an original formulation of cable tension, which reveals a softening behavior when strains become large. The dynamic modulus of cable elasticity is experimentally identified through Dynamic Mechanical Analysis (DMA). Numerical investigations carried out on suspended CDPRs with different sizes show the effect of the proposed tension formulation on the dynamic response of the end-effector.
\end{abstract}

\section{Introduction}

Cable-Driven Parallel Robots (CDPRs), knowing for their large workspace, high dynamics, low cost and the facility of assembling and dissembling, are a class of parallel robots that are actuated by flexible cables. Accordingly, CDPRs are suitable for many applications such as: Rehabilitation [1,2], rescue operations [3], virtual sport machines [4], pick and place 
CDPRs are composed of a moving end-effector, a fixed frame, winches and cables linking the fixed frame to the endeffector. Two configurations of CDPRs are defined according to the number of cables and spatial layout: fully-constrained configuration $[7,8]$ and suspended configuration $[9,10]$.

The positioning accuracy of CDPRs can be significantly degraded because of vibrations in long cables. The stiffness analysis thus becomes a vital concern to improve the static and dynamic behaviors of CDPRs. Considering the physical cable characteristics, the compliance of cables has mainly two origins. The first one is the axial stiffness of the cables, which is associated with the elastic material modulus and the cable structure. The other one is the sag-introduced flexibility, which comes from the effect of cable weight onto the static cable profile. The sag-introduced flexibility corresponds to the gravitational potential energy stored in the cable.

Both axial and sag-introduced stiffness are discussed in the static and oscillatory modeling of CDPRs. Most of the existing elasto-geometrical models of CDPRs deal with the axial stiffness only by considering massless cables [11,12]. Other works in the literature consider inextensible cables with non-negligible mass $[13,14]$ based on the well-known catenary cable model established by Irvine [15]. For instance, such a cable model has been used for the static stiffness analysis of CDPRs and validated experimentally in [16]. Vibration analysis in both directions were made and it turns out that the transversal vibrations remain negligible with respect to the axial vibrations for several configurations [17]. In addition, an oscillatory analysis of a 6-DOF cable-suspended parallel manipulator was established while considering the dynamic stiffness of elastic sagging cables and validation experiments were made in $[16,18]$.

Few research works consider the sag-induced stiffness in the dynamic and workspace analysis of CDPRs. The cables are often considered either rigid or elastic, namely, with an axial stiffness [19]. Simplified cable models are usually used because they lead to lower computing-time [20]. However, those simplified models may lead to modeling errors. These errors should be analyzed and corrected to satisfy the positioning accuracy of the robot. To better understand the dynamic behavior of CDPRs with long cables, a dynamic model where each cable is considered with distributed mass and time-varying length is addressed in [21]. The dynamic equations are derived using Hamilton principle. The resulting infinite dimensional problem is transformed into a finite dimensional one using the assumed-mode method. There are few studies in the literature about the dynamic analysis of CDPRs with long cables, and to the best of our knowledge only cable linear axial stiffness formulation is considered. The dynamic stiffness analysis of CDPRs is made under the assumption that dynamic loads induce only small elongations of the cables. The cable tension is usually considered proportional to the variations in the cable length for a constant stiffness coefficient. Therefore, such a model is not valid when cables are subjected to high strains due to large dynamic oscillations or quick cable-length variations. As a consequence, this paper aims at expressing dynamic and oscillatory motions of CDPRs with cables subjected to fast varying lengths using a non-linear cable tension formulation [22]. This formulation reveals a softening behavior when strains become large.

No matter the constitutive formulation used to describe the stiffness behavior of the cable, the identification of the mechanical parameters of the cable remains a practical issue. Under these conditions, a procedure to identify experimentally the dynamic modulus of cable elasticity is then presented. Dynamical Mechanical Analysis (DMA) is used to study the effect loading frequency on the elastic and damping mechanical properties of a steel wire cable. The sensitivity of the dynamic CDPR responses to the identified modulus is then presented.

This paper is organized as follows. Section 2 expresses the non-linear tension formulation, which is used for dynamic modeling of CDPRs. The dynamic model of a CDPR with three cables and a point-mass end-effector is studied as an illustrative example of the proposed modeling method. Section 3 presents the proposed method to identify the dynamic mechanical parameters of steel wire cables. Section 4 presents the obtained results and analysis on the illustrative example. In order to highlight the contributions of the paper, two CDPRs from the literature, namely the CAROCA prototype [6,23] and the FAST robot [24,25], are studied. The effect of the proposed dynamic modeling onto the pose accuracy of their end-effector is analyzed. Some discussions and conclusions are given in Section 5.

\section{CDPR dynamic modeling based on a non-linear tension formulation}

50 This section deals with the dynamic modeling of a suspended CDPR whose cable sagging is neglected and axial cable vibrations only are considered. This modeling is based on a non-linear cable tension formulation, which is suitable for large strain conditions [22].

\subsection{Non-linear tension formulation}

The total time-depending tension in a cable is classically expressed as follows:

$$
T(t)=k r(t)+T_{0},
$$

where: 
- $T(t)$ is the cable tension,

- $r(t)$ is its elongation,

- $k(t)=\frac{E S}{l(t)}$ is its stiffness,

- $E$ is its modulus of elasticity,

- $S$ is its cross sectional area,

- $l(t)$ is its length,

- $T_{0}$ is its tension at the initial time $(t=0 \mathrm{~s})$.

This linear formulation of the cable tension is commonly used and it shows a behavior similar to a linear spring with constant stiffness $k$. The classical linear tension formulation expressed in Eq. (1) can not satisfy non-linear cable elasticity or viscosity resulting from dynamic phenomena with high cable-length variations. As a result, it cannot be used for the dynamic modeling of CDPR achieving high velocities and accelerations. Therefore, a non-linear tension formulation is required in such a case. The work presented in [22] is adopted regarding that it provides a total non-linear tension formulation valid for high dynamic applications. Accordingly, this paper aims to integrate this new tension formulation in CDPR dynamic modeling and analyze its effect on the dynamic responses of the CDPR end-effector.

According to [22], the non-linear formulation is defined as follows. Applying the general Hooke's law $\sigma(t)=E \varepsilon(t)$, where $d \varepsilon(t)=\frac{d l(t)}{l(t)}$, and assuming that cable tension, with constant cross sectional area $S$, can be written as $T(t)=S \sigma(t)$, the time derivative of the cable tension can be expressed as:

$$
\dot{T}(t)=\frac{E S}{l(t)} \dot{l}(t) .
$$

Equation 2 shows that the time derivative of cable tension depends on the cable instantaneous length. The non-linear tension formulation of the cable tension is obtained by integrating Eq. (2):

$$
T(t)=E S \ln \left(\frac{l(t)}{l_{0}}\right)+T_{0}
$$

This non-linear tension expression is valid for high dynamic systems and does not restrict the applications with small strains. As the cable is considered without inertia, the longitudinal wave propagation is possible and a non-linear cable behavior exists. This non-linear tension formulation is valid when the rate of strain is much smaller than the longitudinal wave velocity into the cable, which is usually true for flexible wires used in high dynamic cable systems and particularly CDPRs.

The dynamic modeling of CDPRs will be based on the non-linear tension formulation expressed in Eq. (3) to study configurations where cables are subjected to high strains and large elongations.

\subsection{CDPR dynamic modeling}

This section aims to establish the dynamic model of a suspended spatial CDPR with three cables and three translational DOF (Fig.1), using the non-linear tension formulation. It is a suspended cable-driven parallel robot where the cable mass is neglected and cables keep a linear profile. As a matter of fact, heavy cables give rise to sagging problems. That leads to non-linearities on the dynamic model of the robot and makes it more complex. The CDPR configuration is chosen such that the cables are tensed along a prescribed trajectory and their tension remains in an elastic range. Moreover, the sag to span ratio of the cable remains lower than $1 / 20$ [26].

The robot cables are fixed to the end-effector and driven by three motors. The cables velocities at pulleys entrances are equal to $V_{1}, V_{2}$ and $V_{3}$, respectively. The $i$ th cable is attached to the fixed platform at point $B_{i}=\left[B_{i x}, B_{i y}, B_{i z}\right]^{T},(i=1,2,3)$. The radius of the pulleys is supposed to be negligible with respect to the overall size of the CDPR. The end-effector is a point mass denoted as $P$.

The $i$ th cable length as a function of time is expressed as follows:

$$
l_{i}(t)=l_{i, 0}+r_{i}(t)-\int_{0}^{t} V_{i}(t) d t, \quad i=1,2,3
$$

where $V_{i}(t)$ is the cable linear velocity at the $i$ th pulley entrance and $l_{i, 0}$ is the $i$ th cable length at time $t=0 \mathrm{~s}$ under the initial tension $T_{i, 0}$ defined with respect to the static equilibrium. 


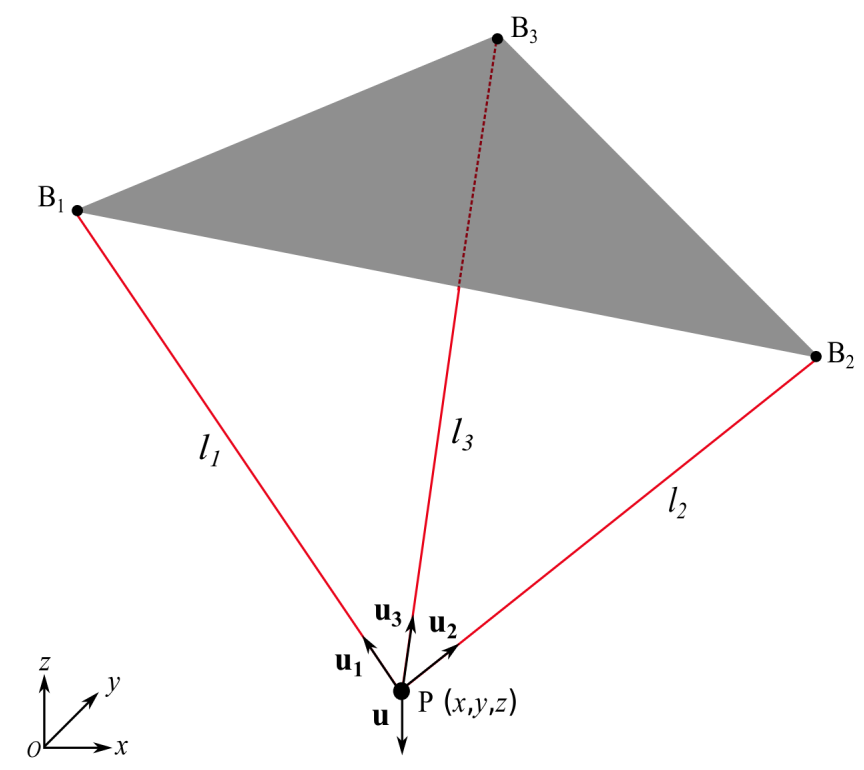

Fig. 1: Schematic diagram of the studied CDPR (3-DOF CDPR suspended by 3 cables)

The motion equations can be obtained by applying the Fundamental Principle of Dynamics on the end-effector of mass $m$ as follows:

$$
m \ddot{\mathbf{p}}=\sum_{i=1}^{3} T_{i} \mathbf{u}_{\mathbf{i}}+m g \mathbf{u}
$$

where $\ddot{\mathbf{p}}=[\ddot{x}, \ddot{y}, \ddot{z}]^{T}$ is the Cartesian acceleration vector of the end-effector, $T_{i}$ is the norm of the tension vector of cable $i$ and $\mathbf{u}_{\mathbf{i}}$ is its unit vector. $\mathbf{u}$ is the unit vector along the direction of $z$-axis and $g$ is the acceleration due to gravity. The time notations are removed from the dynamic equations for a better readability. The cables tensions, lengths and elongations are always time-varying.

The inverse kinematics relationships should be respected. They consist in computing the norm of the vector linking the exit point of the fixed frame from which the cable extends, to the anchor point of the cable where it is attached to the mobile platform. They are expressed as follows:

$$
l_{i}=\sqrt{\left(x-x_{i}\right)^{2}+\left(y-y_{i}\right)^{2}+\left(z-z_{i}\right)^{2}}, \quad i=1,2,3
$$

where $\mathbf{p}=[x, y, z]^{T}$ is the Cartesian coordinates vector of the end-effector and $\mathbf{b}_{i}=\left[x_{i}, y_{i}, z_{i}\right]^{T}$ is the Cartesian coordinates vector of point $B_{i}$.

\section{Steel wire cable: Identification of elasticity modulus 3.1 Modulus of elasticity}

This section aims to specify the identification method of the modulus of elasticity of a steel wire cable used in the cable tension expression. The elasticity modulus or Young's modulus is defined as the ratio of stress along an axis to strain along that 
axis within a specified load range and strain rate range. It amounts to an elastic material stiffness. The modulus of elasticity of a wire cable does not depend solely on the properties of the wire material, but on the wires layout and the load history too. The determination of the modulus of elasticity of a steel wire cable is described in the International Standard ISO 12076.

The cable used in this application consists of 18 strands twisted around a steel core. Each strand is made up of 7 steel wires (Fig. 2a). The breaking force of this cable is $10.29 \mathrm{kN}$. Force controlled cycles from 0 to $3087 \mathrm{~N}$ ( $30 \%$ of the breaking force as specified in the International Standard ISO 12076) were applied 50 times at the rate of $0.05 \mathrm{~mm} / \mathrm{s}$. The free length of cable between the grips of the tensile machine (Fig. 2b) is $350 \mathrm{~mm}$ (15 times higher than the nominal cable diameter). A wire cable should always be loaded and relieved multiple times for identifying the modulus of elasticity. A relative steady state is reached when repeated extension readings are consistent at both ends of the force range. Figure 3 shows the load-elongation diagram of a rotation-resistant steel wire cable of $4 \mathrm{~mm}$ diameter obtained after 50 cycles.

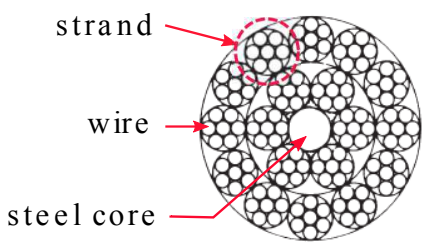

(a)

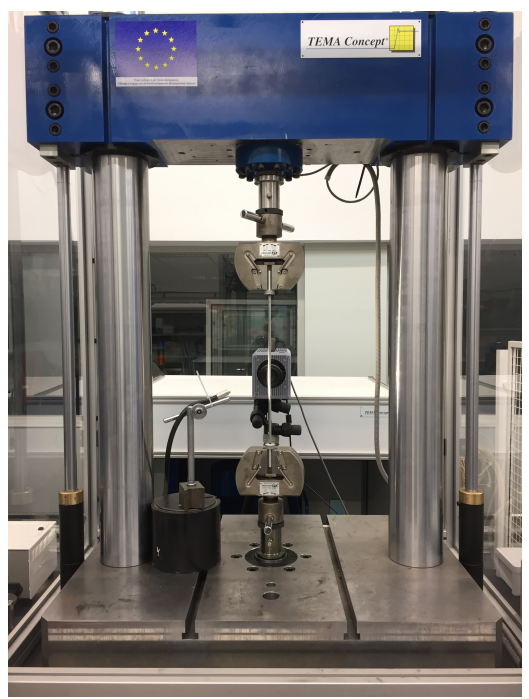

(b)

Fig. 2: Experimental setup: (a) Cross section of a rotation-resistant steel wire cable ; Carl Stahl Technocables Ref 1692 ; (b) Thema Concept cyclic loading test bench

Figure 3 shows an elastic hysteresis loop where the unloading path does not correspond to the loading path. The area in the center of the hysteresis loop is the energy dissipated due to internal friction in the cable. Figure 3 depicts a non-linear correlation in the lower area between load and cable elongation. The load-elongation diagram only gets linear when all the wires in each strand and all the strands in the cable share the load together. According to the International Standard ISO 12076 , the modulus of elasticity of a steel wire cable has to be calculated using the tests reading as follows:

$$
E_{10-30}=l_{c} \frac{F_{30 \%}-F_{10 \%}}{A_{c}\left(x_{2}-x_{1}\right)},
$$

where $A_{c}$ is the metallic cross-sectional area, i.e. the value obtained from the sum of the metallic cross-sectional areas of the individual wires in the rope based on their nominal diameters. $x_{1}$ and $x_{2}$ are the elongations at forces equivalent to $10 \%$ and $30 \%\left(F_{10 \%}\right.$ and $\left.F_{30 \%}\right)$, respectively, of the nominal breaking force of the cable $(10.29 \mathrm{kN})$ measured during the loading path (Fig. 3). $l_{c}$ is the measured initial cable length.

The absolute uncertainty associated with applied force and resulting elongation measurements from the test bench outputs is estimated to be $\pm 1 \mathrm{~N}$ and $\pm 0.03 \mathrm{~mm}$, respectively. The resulting modulus value calculated during a loading in steady state condition is $102.2 \pm 2.2 \mathrm{GPa}$. It is noteworthy that this value differs from the one classically calculated using the cross-sectional area of the cable as a whole, i.e. $S_{c}=\pi\left(\frac{\phi_{c}}{2}\right)^{2} \cdot \phi_{c}$ being the apparent diameter of the cable. In that case the 


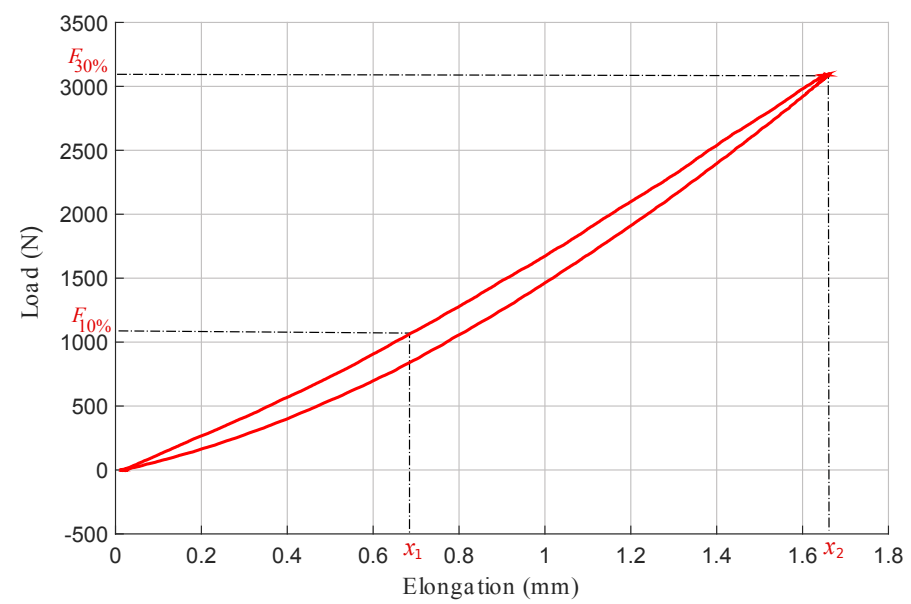

Fig. 3: Load-Elongation diagram of a steel wire cable measured in steady state conditions at the rate of $0.05 \mathrm{~mm} / \mathrm{s}$

modulus of elasticity is equal to $58.2 \pm 1.3 \mathrm{GPa}$. This value differs from the one obtained following the International Standard ISO 12076. The consequences will be limited in the presented application if the definition of the cross-sectional area is the same in the identification procedure of the cable behavior as in the tension expression used in the robot dynamic modeling (Eq. (3)). However, vagueness in data exchange may lead to major modeling errors.

\subsection{Dynamic modulus of elasticity}

Most studies use the modulus of elasticity identified in quasi-static to analyze the dynamic behavior of CDPR [27] [28]. In [29], an original but complex cable model consisting of a rate independent hysteresis model and linear damper is proposed for control of cable-driven mechanisms. In [30], an improved cable model integrating hysteresis effects during force computation was proposed. Here, we propose to use the Dynamic Mechanical Analysis (DMA) to identify the cable stiffness under forced oscillatory measurements [31].

The DMA supplies a sinusoidal load to be applied to the cable, which generates a sinusoidal elongation. By measuring both the amplitude of the resulting elongation and the lag between the force and the elongation, the modulus of elasticity and also the damping of the cable can be identified. One advantage of DMA is that we can obtain a dynamic modulus for different frequencies of the sine wave applied, allowing us to sweep across the frequency range of the intended application. Six tests were performed on the cable at frequencies of $0.1,1,2,5,10$, and $20 \mathrm{~Hz}$ (Fig. 4). A preload was applied to the cable before the sinusoidal force controlled waves, at $1500 \mathrm{~N}$ corresponding to an operating point in the linear area of the load-elongation diagram.

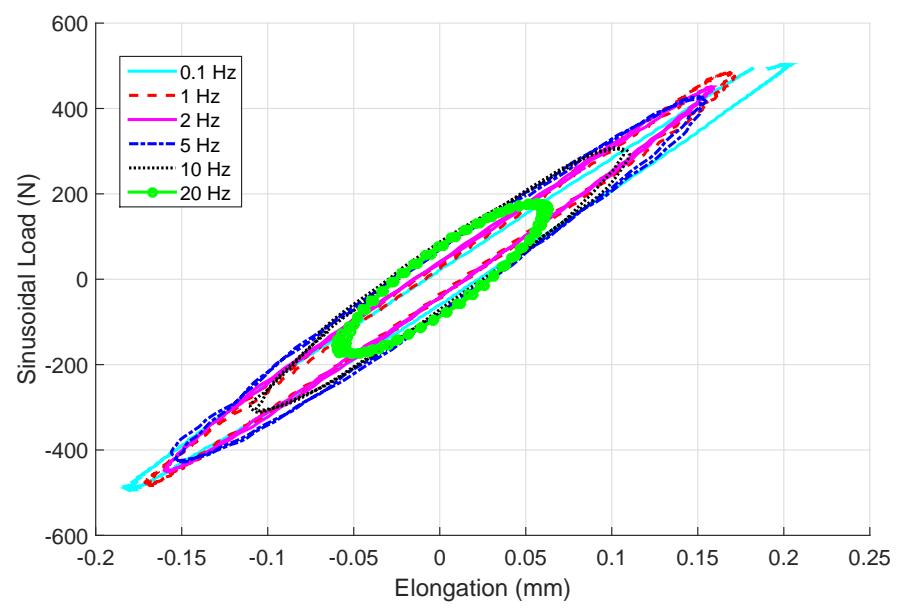

Fig. 4: Hysteresis loops for a $4 \mathrm{~mm}$ steel wire cable preloaded at $1500 \mathrm{~N}$ with force controlled sine waves applied at $0.1,1,2$, 5,10 , and $20 \mathrm{~Hz}$ 
The DMA allows us to calculate from the cable response to the sine wave a dynamic modulus $E^{*}$, which is a complex value. The real part of the dynamic modulus $E^{\prime}$ represents the ability of the cable to return energy and can be assimilated to the elastic modulus. The imaginary part $E^{\prime \prime}$ provides the capacity of the cable to dissipate energy. The applied sinusoidal force and resulting elongation are used to calculate the absolute value of the dynamic modulus $\left|E^{*}\right|$, i.e. the ratio of the maximum amplitude of the applied idealized stress $\sigma_{\max }$ to the maximum amplitude of the resulting strain $\varepsilon_{\max }$, as well as the phase angle $\varphi$ between the force and elongation. In this paper, the definition of the strain assumes an idealized stress-strain response with a constant metallic cross-sectional area of the individual wires in the cable, based on their nominal diameters. The corresponding elastic, $E^{\prime}$, and loss, $E^{\prime \prime}$, moduli are obtained from the dynamic modulus and phase angle as $E^{\prime}=\left|E^{*}\right| \cdot \cos \varphi$ and $E^{\prime \prime}=\left|E^{*}\right| \cdot \sin \varphi$ [32]. The intrinsic damping of the cable can be described by the loss factor [33]:

$$
\eta=\frac{E^{\prime \prime}}{E^{\prime}}=\tan (\varphi)
$$

which is the ratio between the imaginary and real parts of the complex modulus. The dynamic and elastic modulus, the phase angle $\varphi$ and the loss factor $\eta$ are given for each DMA test in Table 1.

Table 1: Frequency dependency of the modulus of elasticity of the steel wire cable at loading frequencies between 0.1 and $20 \mathrm{~Hz}$

\begin{tabular}{c|ccccccc}
\hline \hline Frequencies $(\mathrm{Hz})$ & $\sigma_{\max }(\mathrm{MPa})$ & $\varepsilon_{\max }$ & $\left|E^{*}\right|(\mathrm{GPa})$ & $E^{\prime}(\mathrm{GPa})$ & $\varphi\left(^{o}\right)$ & $\eta$ & $c\left(N . m^{-1} . s\right)$ \\
\hline 0.1 & 69.731 & $5.743910^{-4}$ & $121.4 \pm 2.8$ & $120.5 \pm 2.8$ & $6.8 \pm 2.3$ & $0.12 \pm 0.04$ & $22.810^{9}$ \\
1 & 67.136 & $4.847410^{-4}$ & $138.5 \pm 3.3$ & $137.3 \pm 3.3$ & $7.5 \pm 2.8$ & $0.13 \pm 0.05$ & $2.8710^{9}$ \\
2 & 62.379 & $4.549910^{-4}$ & $137.1 \pm 3.3$ & $134.8 \pm 3.1$ & $11.0 \pm 2.8$ & $0.19 \pm 0.05$ & $2.0810^{9}$ \\
5 & 58.654 & $4.315910^{-4}$ & $135.9 \pm 3.1$ & $131.0 \pm 3.0$ & $14.1 \pm 2.8$ & $0.25 \pm 0.05$ & $1.0410^{9}$ \\
10 & 41.618 & $3.049010^{-4}$ & $136.5 \pm 3.2$ & $130.1 \pm 3.0$ & $17.6 \pm 2.8$ & $0.31 \pm 0.05$ & $0.6510^{9}$ \\
20 & 24.025 & $1.744710^{-4}$ & $137.7 \pm 3.3$ & $126.2 \pm 2.8$ & $23.5 \pm 2.8$ & $0.43 \pm 0.05$ & $0.4410^{9}$ \\
\hline \hline
\end{tabular}

We can see that the stiffness and damping are highly dependent on frequency for a given preload, over a representative frequency range of the CDPR behavior [16]. The elastic modulus increases significantly at very low frequencies, from $120.5 \pm 2.8 \mathrm{GPa}$ at $0.1 \mathrm{~Hz}$ to $137.3 \pm 3.3 \mathrm{GPa}$ at $1 \mathrm{~Hz}$ (Table 1 ). This is compared to the quasi-static modulus of elasticity calculated following the International Standard whose value is equal to $102.2 \pm 2.2 \mathrm{GPa}$. The elastic modulus is secondly found to decrease slowly with higher frequency. At higher frequencies, the dynamic modulus is approximately constant, with values within the confidence interval referring to the reliability of the test procedure. Large changes in the phase angle occur over the frequency range. The frequency-dependent loss factor appears consistent with increased hysteresis and thus loading velocity.

The cable damping is modeled by using a linearly viscoelastic definition. Indeed, an equivalent viscous coefficient can be defined as:

$$
c=\frac{E^{\prime} \eta}{2 \pi f}
$$

$f$ being the loading frequency.

These dynamic characteristics indicate how well the cable works in oscillatory motions around a static equilibrium or along a dynamic trajectory since we can get this value for a range of frequencies. Our results show that the apparent cable stiffness can change significantly under dynamic loading and may lead to large displacements of the CDPR end-effector.

\subsection{Non-linear tension formulation of cables with linear damper}

The previous part shows that the cables used for the CDPR have a frequency-dependent dynamic modulus of elasticity. To better predict the dynamic of cables and the CDPR response, we can account for elastic cables with linear viscous damper 
model. Damping parameters are extracted from the previous part. For an elastic cable with a linear viscous damper, the cable stress is written as follows:

$$
\sigma(t)=E^{\prime} \varepsilon(t)+c \dot{\varepsilon}(t)
$$

where $E^{\prime}$ is the real part of the dynamic modulus of elasticity and $c$ is the correspondent damping coefficient.

As $T(t)=S \sigma(t)$ for a constant cross sectional area cable and as the differentiation of deformation $\dot{\varepsilon}(t)$ is expressed as $\frac{i(t)}{l(t)}$, we can write:

$$
\dot{T}(t)=\frac{E^{\prime} S}{l(t)} \dot{l}(t)+\frac{c S}{l(t)}\left(\ddot{l}(t)-\frac{\dot{l}^{2}(t)}{l(t)}\right)
$$

By integrating Eq. (11), the new tension formulation considering the cable damping is defined as follows:

$$
T(t)=E^{\prime} S \ln \left(\frac{l(t)}{l_{0}}\right)+c S \frac{i(t)}{l(t)}+T_{0}
$$

This formulation considers the non-linear elastic behavior of the cable and its damping. It will be valid even if the system

\subsubsection{Illustrative example of 3-DOF and 3 cables}

This section aims to present numerical results associated to the dynamic behavior of the 3-DOF CDPR proposed in Section 2, where three long cables make the end-effector move. The position vector of the exit points in the global frame are: $\mathbf{b}_{1}=[0,50,50]^{T} \mathrm{~m}, \mathbf{b}_{2}=[0,-50,50]^{T} \mathrm{~m}$ and $\mathbf{b}_{3}=[86.60,0,50]^{T} \mathrm{~m}$. The mass of the end-effector is $m=300 \mathrm{~kg}$.

An example of a circular helical trajectory from static equilibrium to steady state is firstly proposed to evaluate the difference between the linear and non-linear tension formulations. The end-effector of the CDPR under study is moved from point $P_{1}$ of Cartesian coordinate vector $\mathbf{p}_{1}=[28.86,0,5]^{T} \mathrm{~m}$ to point $P_{2}$ of Cartesian coordinate vector $\mathbf{p}_{2}=[28.86,0,20]^{T} \mathrm{~m}$ tracking a circular helix, which is defined by the following Cartesian equations (Fig. 5a):

$$
\begin{aligned}
& x(t)=R \cos \left(t_{\alpha}\right)+\beta_{0}, \\
& y(t)=R \sin \left(t_{\alpha}\right)+\beta_{1}, \\
& z(t)=p t_{\alpha}+\beta_{2},
\end{aligned}
$$

where $t_{\alpha}=a_{5}\left(\frac{t}{t_{\text {sim }}}\right)^{5}+a_{4}\left(\frac{t}{t_{\text {sim }}}\right)^{4}+a_{3}\left(\frac{t}{t_{\text {sim }}}\right)^{3}+a_{2}\left(\frac{t}{t_{\text {sim }}}\right)^{2}+a_{1}\left(\frac{t}{t_{\text {sim }}}\right)+a_{0}$. The coefficients of the five-order polynomial $t_{\alpha}$ are chosen in such a way that the Cartesian velocities are null at the beginning and the end of the simulation (Fig. 
5b). $R$ is the radius of the helix, $p$ is its pitch, $\beta_{0}, \beta_{1}$ and $\beta_{2}$ are constants. The resulting Cartesian velocities of the end-effector are expressed as follows:

$$
\begin{aligned}
\dot{x}(t) & =-R \dot{t}_{\alpha} \sin \left(t_{\alpha}\right), \\
\dot{y}(t) & =R \dot{t}_{\alpha} \cos \left(t_{\alpha}\right), \\
\dot{z}(t) & =p \dot{t}_{\alpha} .
\end{aligned}
$$

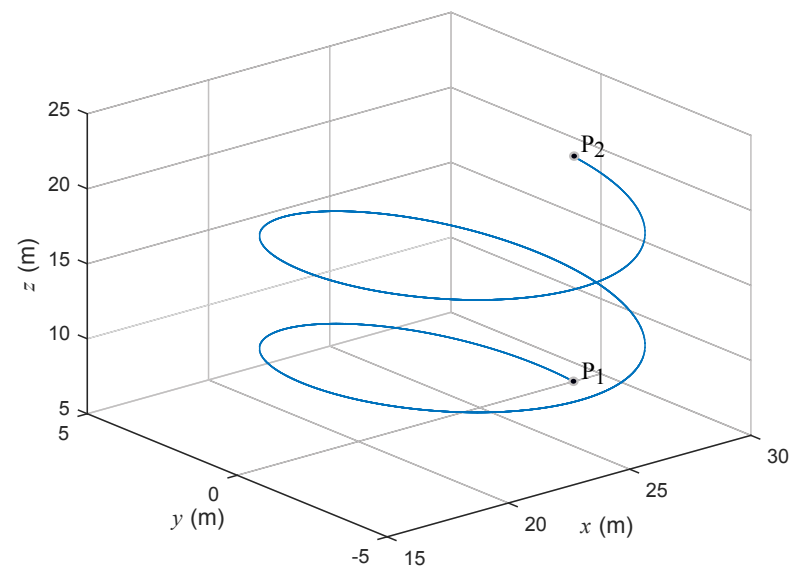

(a) End-effector trajectory

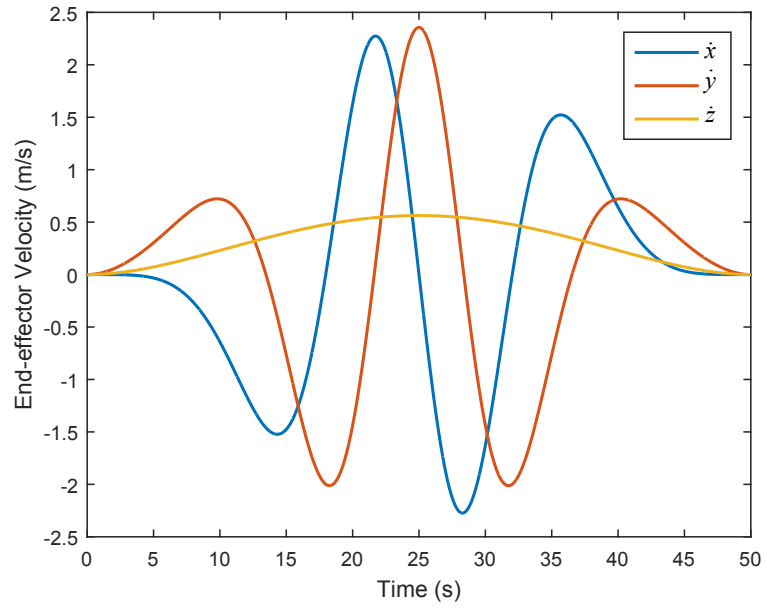

(b) End-effector Cartesian velocities

Fig. 5: Circular helical trajectory

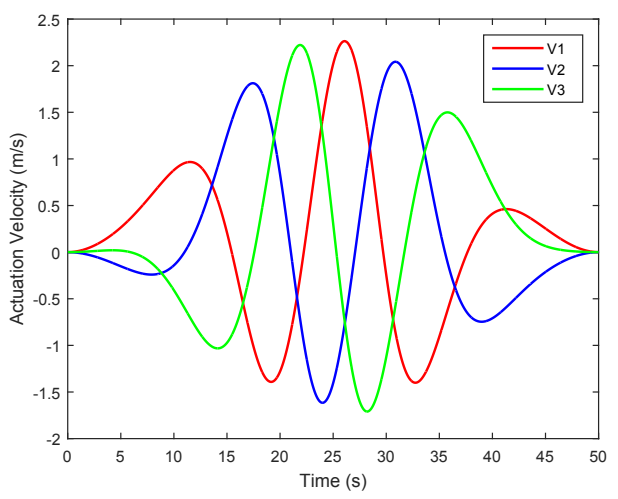

Fig. 6: Cable linear velocity profiles

For this case study, the chosen parameters are: $a_{5}=36 \pi, a_{4}=-90 \pi, a_{3}=60 \pi, a_{2}=a_{1}=a_{0}=0, p=0.5 \mathrm{~m}$, $\beta_{0}=23.86 \mathrm{~m}, \beta_{1}=0 \mathrm{~m}, \beta_{2}=5 \mathrm{~m}, R=5 \mathrm{~m}$ and $t_{\text {sim }}=50 \mathrm{~s}$. The initial cables lengths are $l_{1,0}=l_{2,0}=l_{3,0}=73.2 \mathrm{~m}$. The simulation starts from the static equilibrium defined with the following initial conditions: $T_{1,0}=T_{2,0}=T_{3,0}=1594.1 \mathrm{~N}$, $\dot{r}_{1,0}=\dot{r}_{2,0}=\dot{r}_{3,0}=0 \mathrm{~m} / \mathrm{s}$ and $\ddot{r}_{1,0}=\ddot{r}_{2,0}=\ddot{r}_{3,0}=0 \mathrm{~m} / \mathrm{s}^{2}$. Figure 6 illustrates the cable linear velocities as a function of time for the end-effector to follow the desired trajectory shown in Fig. 5a.

CDPR with purely elastic cables will be first simulated to evaluate the difference between linear and non-linear tension formulations. In this simulation, the quasi-static equivalent modulus of elasticity is used. As this modulus is identified while respecting the International Standard ISO 12076, the cross sectional area $S$ should be assimilated to $A_{c}$ and not as a whole 
$\left(S=A_{c} \neq S_{c}\right)$. For $S=A_{c}=7.1675 \times 10^{-6} \mathrm{~m}^{2}$, the quasi-static equivalent modulus of elasticity is $E=102.2 \pm 2.2 \mathrm{GPa}$ as identified experimentally.

The time histories of the tension, the elongation and the strain (defined as $\frac{r(t)}{l(t)}$ ) of each cable when the end-effector moves from point $P_{1}$ to point $P_{2}$ following the circular helical trajectory are plotted in Fig. 7. The comparison between the simulation results obtained with the linear tension (Dash-dot lines) and the non-linear tension formulation (continuous lines) is made. In both cases, the cable tensions remain positive which is a necessary condition for CDPR control. It can also be noticed that the generated trajectory has sufficient smoothness properties to avoid the excitation of the mechanical resonances of the end-effector suspended on the global cables stiffness. The loading frequency is only defined by the time variation of the cable linear velocity (Fig. 6).

These simulation results show that the difference in terms of cable elongation response can reach up to $2.4 \mathrm{~mm}$ at $27.5 \mathrm{~s}$ for the first cable, representing $14 \%$ of the global instantaneous elongation. The difference in terms of strain achieves $0.003 \%$, representing $12 \%$ of the global instantaneous strain in cable 1 . The maximum difference is about $2.9 \mathrm{~mm}$ for the second cable at $39 \mathrm{~s}$. Note that the cable tensions are the same whichever tension formulation is used. Tension curves are superimposed (Figures $7 \mathrm{a}, 7 \mathrm{~d}$ and $7 \mathrm{~g}$ ). The non-linear tension formulation reveals a softening behavior when strains become large. In this particular example of large-dimensions CDPR, the consequences on the evaluation of the end-effector position tracking error can be quite important as depicted in Fig. 8. The positioning error, which is the difference between the desired position of the end-effector and the real one, is assessed along each axis of the base frame. The end-effector trajectory can differ up to mm along the $x$-axis, $1.4 \mathrm{~mm}$ along the $y$-axis and $2.9 \mathrm{~mm}$ along the $z$-axis depending on the formulation. Even if the end-effector position tracking errors are relatively small compared to the overall size of the robot in this particular example, this represents a maximum relative difference of $22 \%$ in the computation of the end-effector tracking errors. This emphasizes the value of the proposed approach for some applications with large dimensions, which improve the tracking accuracy by a model-based compensation [34]. The relevance of the proposed method formulation should be also verified for a CDPR with smaller dimensions. For this purpose, a comparison between the dynamic responses of two existing CDPRs with different sizes is presented thereafter.

\subsubsection{Semi-industrial examples}

The previous example aims to highlight the difference between linear and non linear cable tension formulations. For a better understanding of the effect of the cable tension model onto the moving-plateform pose estimation, two semi-industrial R prototypes are considered in this section: The 6-dof FAST robot [24,25] composed of 6 cables and the CAROCA prototype developed at IRT Jules Verne [6,23] in a configuration such that it contains 3 cables and performs 3-DOF translational motions. Both CDPRs are described in Tab. 2. This latter presents the height, width, the unstrained cables length when the end-effector is in a home position, the end-effector mass and the maximum cable tension.

Table 2: Characteristics of CAROCA and FAST CDPRs

\begin{tabular}{c|ccccc}
\hline \hline & Height $(\mathrm{m})$ & Width $(\mathrm{m})$ & $\begin{array}{c}\text { Unloaded cable } \\
\text { length }(\mathrm{m})\end{array}$ & $\begin{array}{c}\text { End-effector } \\
\text { mass }(\mathrm{kg})\end{array}$ & $\begin{array}{c}\text { Maximal design allowable } \\
\text { cable tension }(\mathrm{kN})\end{array}$ \\
\hline CAROCA & 3.5 & 7 & 4.6 & 100 & 10 \\
\hline \hline FAST & 376 & 500 & 284.7 & 10000 & 500 \\
\hline \hline
\end{tabular}

\section{- CAROCA}

The Cartesian coordinates of the cable exit points for the CAROCA prototype (Fig. 9a) are expressed as: $\mathbf{b}_{1}=[3.5,-2,3.5]^{T}$ $\mathrm{m}, \mathbf{b}_{2}=[0,2,3.5]^{T} \mathrm{~m}$ and $\mathbf{b}_{3}=[-3.5,-2,3.5]^{T} \mathrm{~m}$ into the base frame. The CAROCA end-effector follows an helical trajectory whose parameters are: $a_{5}=3.6 \pi, a_{4}=-9 \pi, a_{3}=6 \pi, a_{2}=a_{1}=a_{0}=0, p=0.2 \mathrm{~m}, \beta_{0}=0 \mathrm{~m}, \beta_{1}=0 \mathrm{~m}$, $\beta_{2}=0.1 \mathrm{~m}, R=1 \mathrm{~m}$ and $t_{\text {sim }}=4 \mathrm{~s}$. The same cables parameters as the previous example are used . For purpose of visibility, only the second cable behavior is plotted (Fig. 10).

The tension, elongation and strain profiles for cable 2 obtained with both the linear and non-linear cable tension models, when the end-effector tracks the helical trajectory going from point $P_{1}$ of Cartesian coordinate vector $\mathbf{p}_{1}=[1,0,1]^{T} \mathrm{~m}$ to point $P_{2}$ of Cartesian coordinate vector $\mathbf{p}_{2}=[-0.4,1,1.37]^{T} \mathrm{~m}$, are plotted in Fig. 10.

These simulation results show that the maximum difference in terms of strain is about $0.0014 \%$, representing $15.06 \%$ of the global instantaneous strain. The difference in terms of cable elongation reaches up to $0.034 \mathrm{~mm}$ for the second cable, 


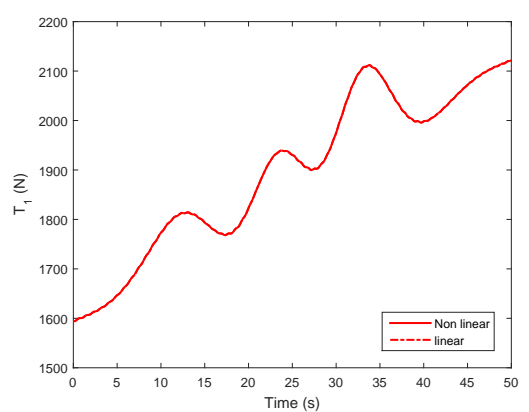

(a) Time history of tension $T_{1}$

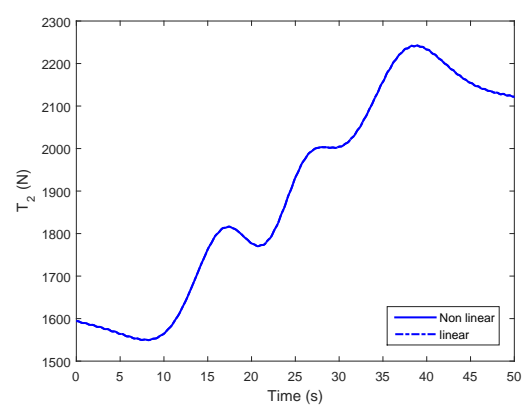

(d) Time history of tension $T_{2}$

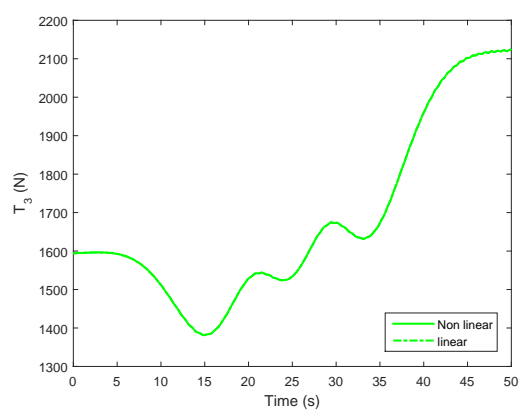

(g) Time history of tension $T_{3}$

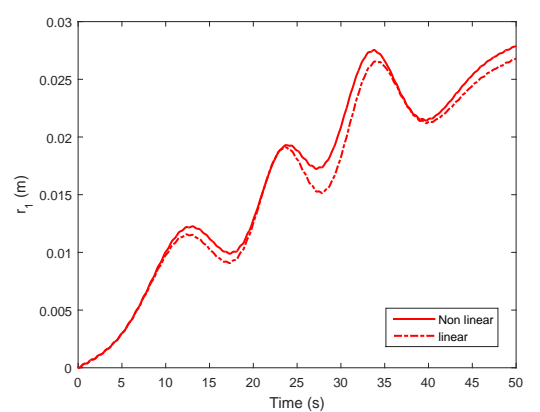

(b) Time history of elongation $r_{1}$

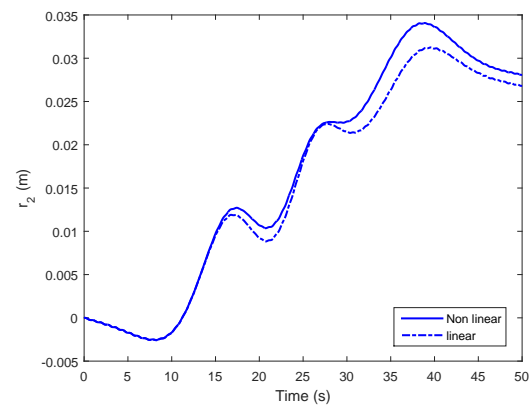

(e) Time history of elongation $r_{2}$

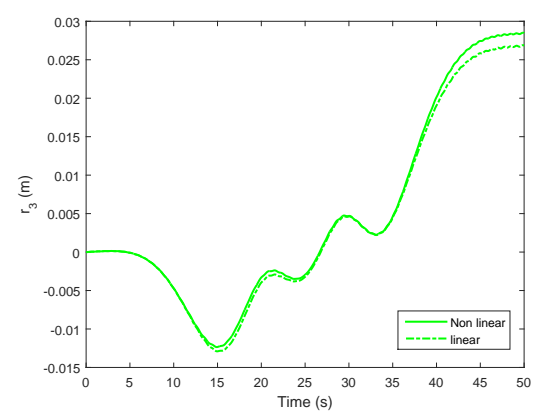

(h) Time history of elongation $r_{3}$

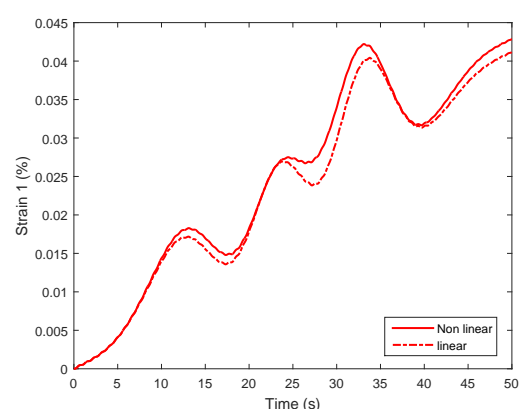

(c) Time history of strain of cable 1

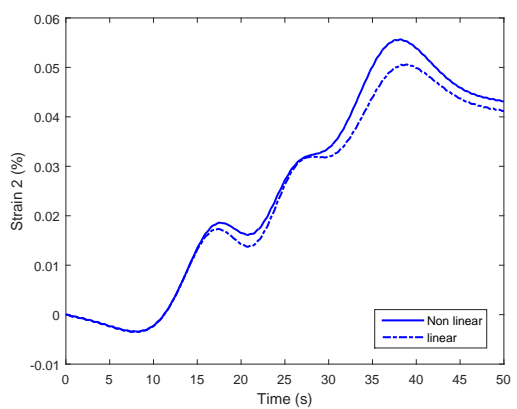

(f) Time history of strain of cable 2

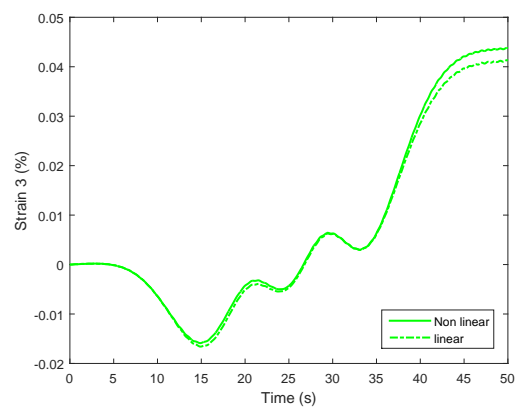

(i) Time history of strain of cable 3

Fig. 7: Comparison between linear and non-linear tension formulations: Tension, elongation and strain

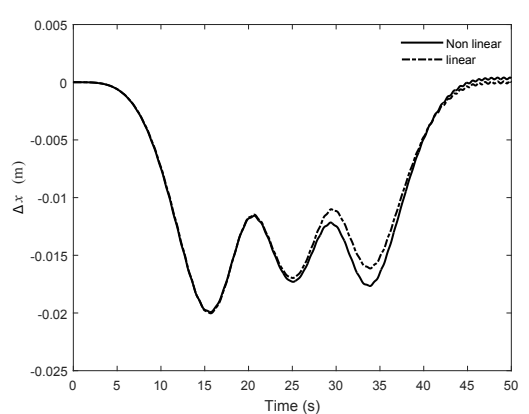

(a) Time history of positioning error along $\mathrm{x}$-axis

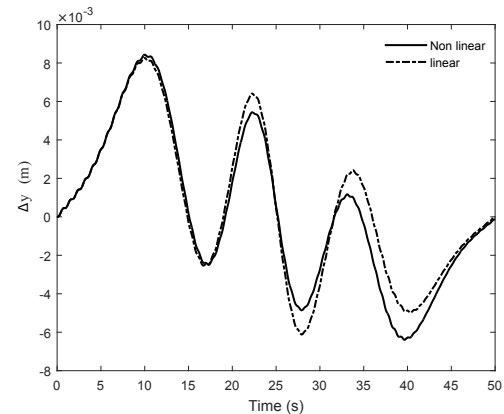

(b) Time history of positioning error along y-axis

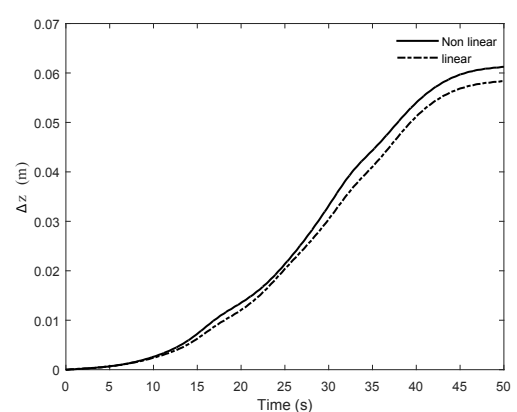

(c) Time history of positioning error along z-axis

Fig. 8: Comparison between linear and non-linear tension formulations: Positioning error of the end-effector along $x$-axis, $y$-axis and $z$-axis 


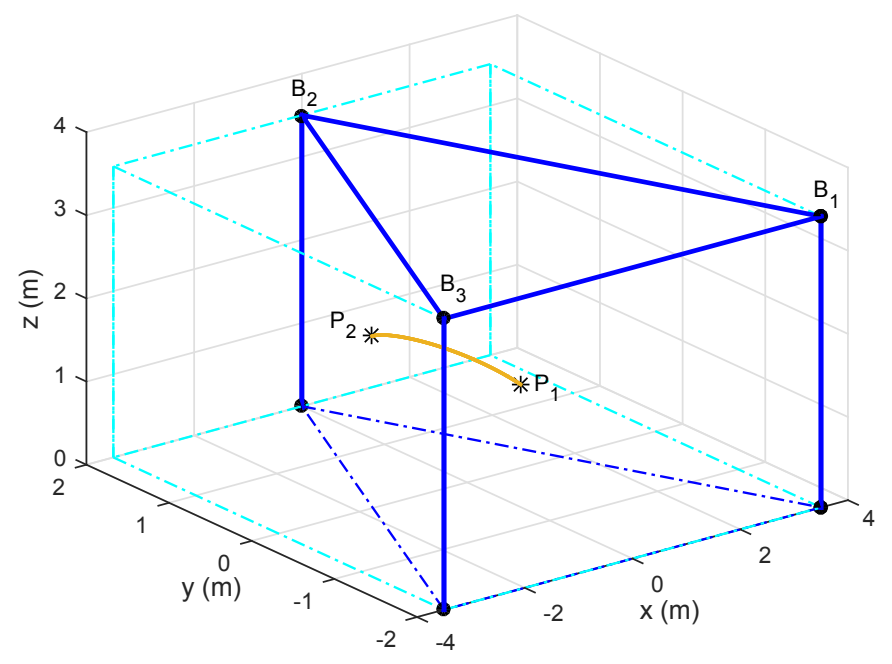

(a) CAROCA

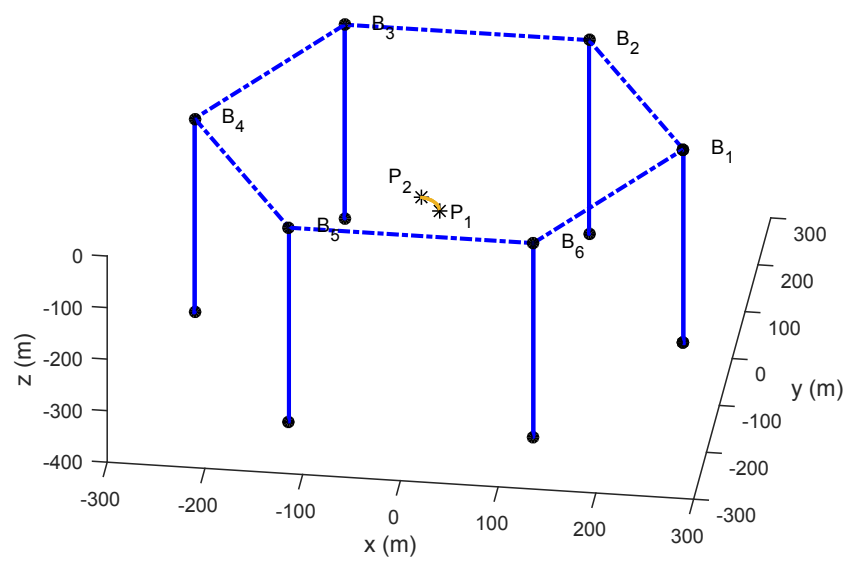

(b) FAST

Fig. 9: Schematics of the (a) CAROCA and (b) FAST CDPR

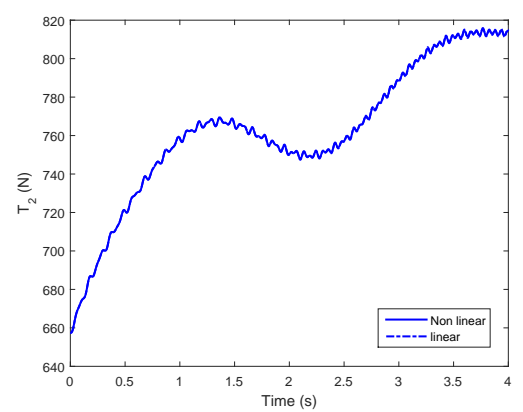

(a) Time history of tension $T_{2}$ : CAROCA

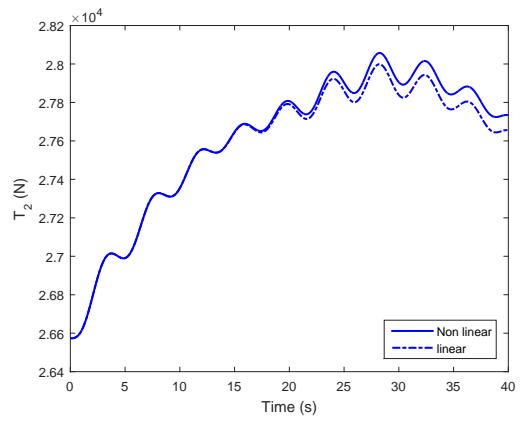

(d) Time history of tension $T_{2}$ : FAST

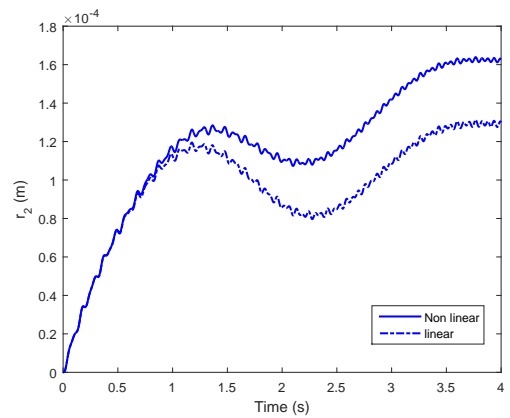

(b) Time history of elongation $r_{2}$ : CAROCA

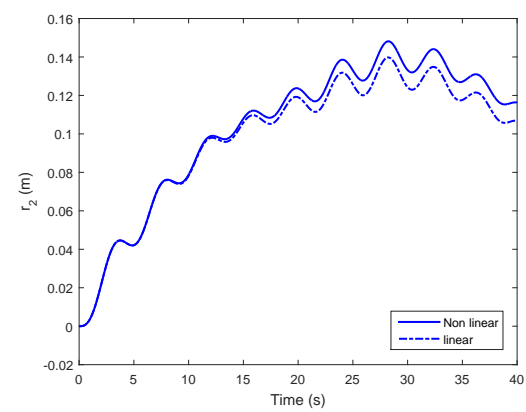

(e) Time history of elongation $r_{2}$ : FAST

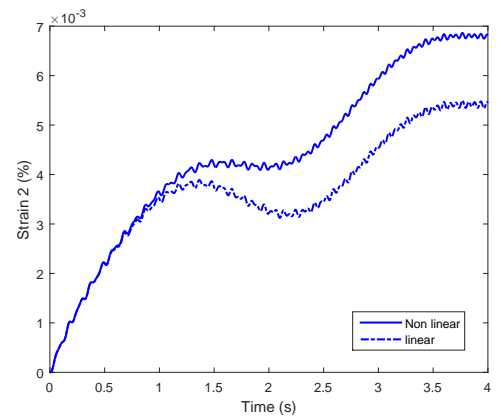

(c) Time history of strain of cable 2: CAROCA

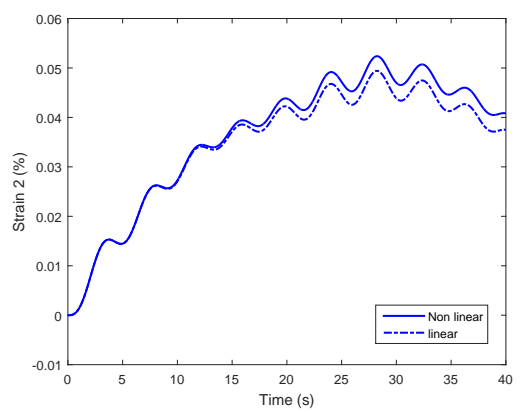

(f) Time history of strain of cable 2: FAST

Fig. 10: Comparison between linear and non-linear tension formulations: Cables responses

representing $25.27 \%$ of the global elongation at $4 \mathrm{~s}$. As shown in Fig. 11, there is a small difference between the positioning error of the end-effector calculated while considering a linear cable tension model and the one estimated by considering a non-linear cable tension model. This difference is about $0.06 \mathrm{~mm}$ and amounts to $6.26 \%$ of the global positioning error of the end-effector. Note that it is difficult to validate those theoretical results experimentally due to the small variations in positioning errors that cannot be measured accurately with a absolute measuring position system such as a laser tracker.

\section{- FAST}

Figure $9 \mathrm{~b}$ represents a schematic of the 6-dof FAST CDPR described in [13]. Its end-effector moves along an helical 


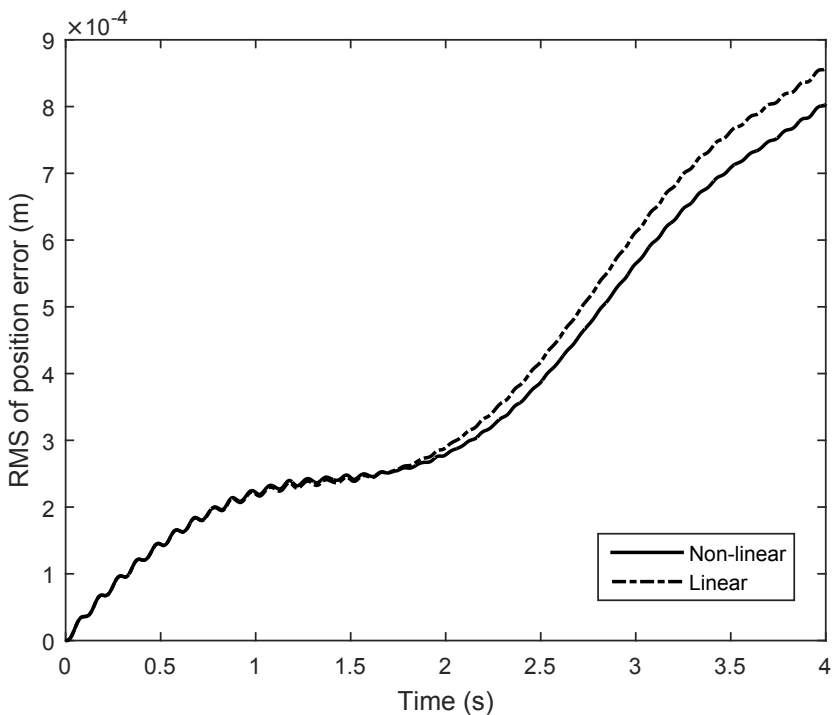

(a) CAROCA

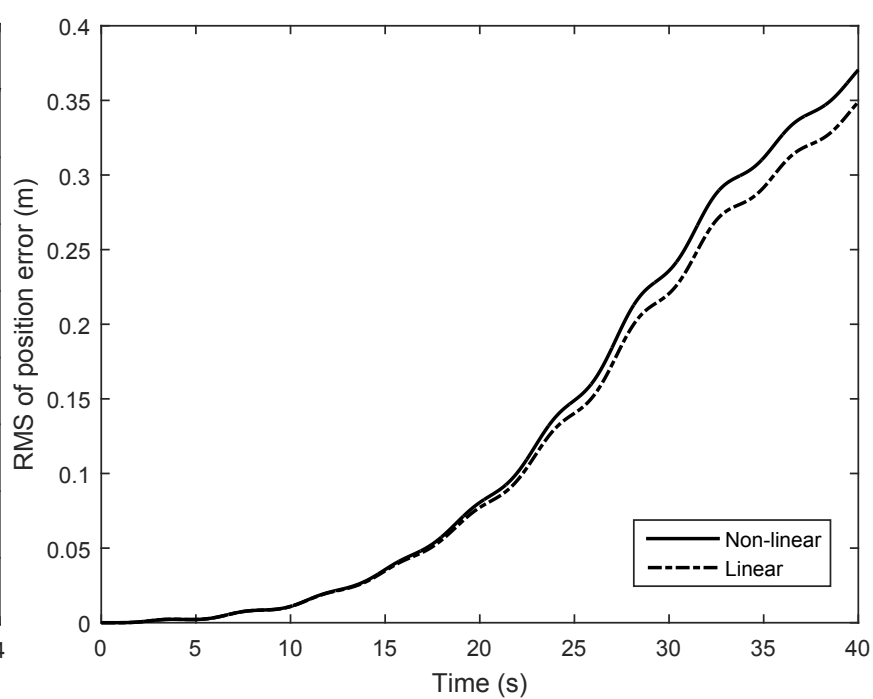

(b) FAST

Fig. 11: Positioning errors of the CDPR end-effector calculated with a linear and a non-linear cable tension model, respectively

path from point $P_{1}$ of Cartesian coordinate vector $\mathbf{p}_{1}=\left[\begin{array}{lll}0, & 0, & -150\end{array}\right]^{T} \mathrm{~m}$ to point $P_{2}$ of Cartesian coordinate vector $\mathbf{p}_{2}=[-19.64,14.27,-138.7]^{T} \mathrm{~m}$ within $t_{\text {sim }}=40 \mathrm{~s}$. The trajectory parameters $a_{0}, a_{1}, a_{2}, a_{3}, a_{4}$ and $a_{5}$ are the same as those used to define the trajectory followed by CAROCA, i.e., $p=6 \mathrm{~m}, R=15 \mathrm{~m}, \beta_{0}=-15 \mathrm{~m}, \beta_{1}=0 \mathrm{~m}$ and $\beta_{2}=-150 \mathrm{~m}$.

Figure 10 represents the time history of the tension, the elongation and the strain profiles for the second cable of the FAST robot by considering a linear and non-linear cable-tension models, respectively. The simulation results show that the difference between the elongations assessed with linear or non-linear cable tension formulations is equal to $8.7 \mathrm{~mm}$ at $38 \mathrm{~s}$, which amounts to $7.2 \%$ of the global instantaneous cable elongation. This difference is due to variation in cable stiffness and leads to a sudden cable tension change (about $71.35 \mathrm{~N}$ ). The maximum difference in terms of cable strain is about $0.0031 \%$, which amounts to $7.42 \%$ of the global instantaneous strain of the second cable. Finally, as shown in Fig. 11, the difference in terms of end-effector positioning error is up to $21.52 \mathrm{~mm}$ at $40 \mathrm{~s}$.

As a conclusion, for very large CDPRs such as the FAST robot, the cable tension model that is considered to estimate the pose error of the end-effector do affect the results. Although the variations in pose error estimation obtained from one cable tension model to the other one remain small with respect to the CDPR size, the non-linear cable tension had better be considered to improve the end-effector trajectory tracking.

\subsection{Dynamic stiffening}

In the previous section, the end-effector performs a smooth and continuous motion limiting the oscillations. Therefore, a trapezoidal-velocity trajectory is chosen to analyze the influence of the cable modulus of elasticity on the CDPR dynamic behavior. The lack of smoothness of the trajectory is chosen here to excite the end-effector on its fundamental rigid-body mode frequencies [16]. The shape of the cable linear velocities at the pulley entrance is defined such as the motion is uniformly accelerated until the linear cable velocities achieve the limit value $V_{\max }$ (Fig. 12a). Linear cable accelerations are defined by the coefficients $a_{1}, a_{2}$ and $a_{3}$ expressed in $\mathrm{m} / \mathrm{s}^{2}$.

To better visualize the effect of the dynamic stiffness on the dynamic and oscillatory motions of the CDPR, the natural frequencies of the cable manipulator are determined by solving the generalized eigenvalue problem associated with the apparent stiffness of the CDPR. Let $f_{1}$ denote the first natural frequency, $f_{2}$ the second natural frequency and $f_{3}$ the third natural frequency.

Both the numerical example and the CAROCA prototype are analyzed to check the effect of the frequency dependency of the dynamic stiffness with respect to the CDPR dimensions.

For the example under study, the coefficients of the cable trapezoidal-velocity profiles are $a_{1}=0.3 \mathrm{~m} / \mathrm{s}^{2}, a_{2}=0.25 \mathrm{~m} / \mathrm{s}^{2}$, $a_{3}=-0.25 \mathrm{~m} / \mathrm{s}^{2}$ and $V_{\max }=1 \mathrm{~m} / \mathrm{s}$. As a result, the manipulator tracks a straight line from point $P_{1}$ of Cartesian coordinate vector $\mathbf{p}_{1}=[28.86,0,5]^{T} \mathrm{~m}$ to point $P_{3}$ of Cartesian coordinate vector $\mathbf{p}_{3}=[20.30,0.23,8.5]^{T} \mathrm{~m}$ in $7 \mathrm{~s}$ (Fig. 12b).

As mentioned in Section 3, DMA allows us to identify the cable's modulus of elasticity under forced oscillatory motions in the frequency range of the intended application. The time histories plotted in Fig. 13 show a resulting forced elongation at a frequency between 1 and $2 \mathrm{~Hz}$, which corresponds to the frequency of the fundamental rigid-body mode of the end-effector 
suspended on the global cable stiffness. Based on the experimental data presented in Tab. 1, the corresponding value of the dynamic modulus of elasticity is about $30 \%$ higher than the value identified in quasi-static. $E^{\prime}=134.8 \mathrm{GPa} \pm 3.1 \mathrm{GPa}$ at $2 \mathrm{~Hz}$ instead of $E=102 \mathrm{GPa} \pm 2.2 \mathrm{GPa}$ in quasi-static.

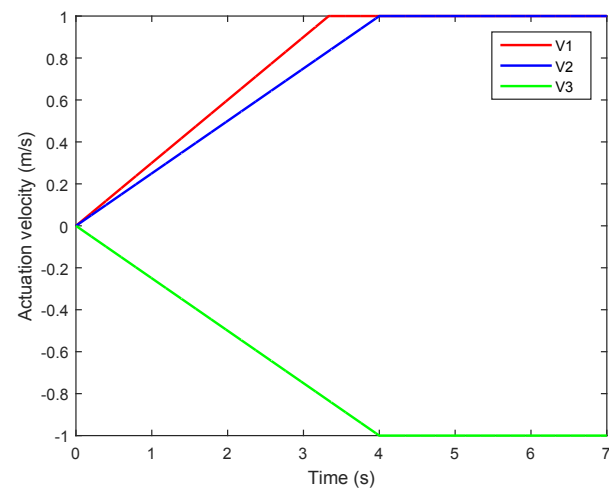

(a) Trapezoidal actuation velocities

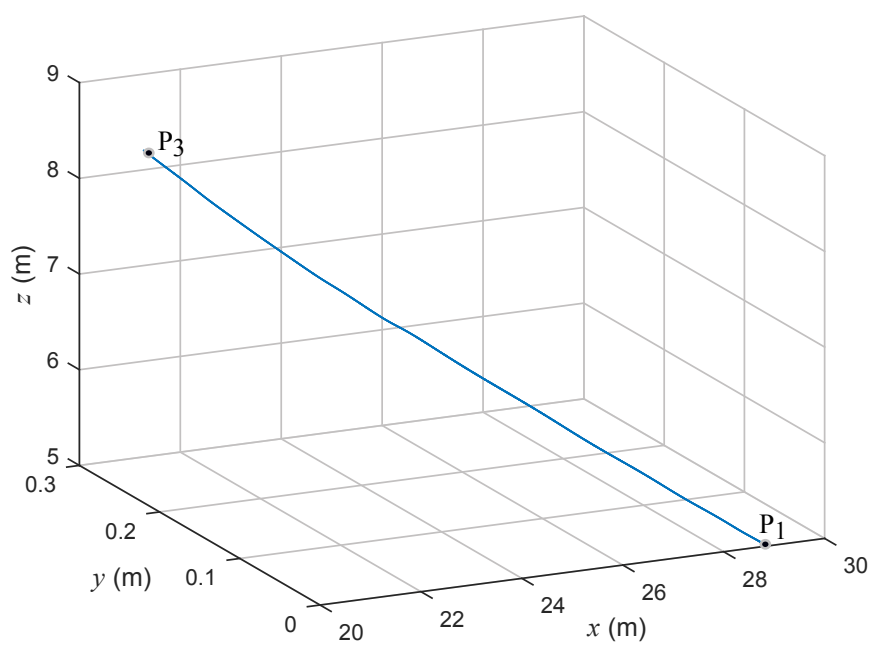

(b) Trajectory of the end-effector

Fig. 12: Trapezoidal-velocity trajectory

Figure 13 shows the tension, the elongation and the strain of the first cable when the value identified in quasi-static at $E=102 \mathrm{GPa}$ (continuous line) or in dynamic at $E^{\prime}=134.8 \mathrm{GPa}$ at $2 \mathrm{~Hz}$ (dashed lines) is used. These curves are computed for the non-linear tension formulation without damping. Since the value of the modulus of elasticity is different, a difference in terms of the oscillation frequency appears in the cable responses. The oscillation frequency increases due to the dynamic stiffening phenomena. This dynamic cable stiffening leads to reduce the elongation amplitude over the trajectory. The differences on the end-effector position tracking error are significant both on the shape of the curves and on their level (Fig. 14). The end-effector trajectory can differ up to $8 \mathrm{~mm}$ along the $x$-axis, $4.5 \mathrm{~mm}$ along the $y$-axis and $9 \mathrm{~mm}$ along the $z$-axis depending on the chosen stiffness. The magnitude of the end-effector positioning errors is significant. Even if these values are relatively small compared to the overall dimensions of the CDPR under study, this represents a maximum relative difference of $24 \%$ in the computation of the end-effector tracking errors.

Figure 15 represents the time variation of the three natural frequencies for different cable stiffnesses. The fundamental natural frequency when the dynamic stiffness is considered is around $1.9 \mathrm{~Hz}$. However, when the quasi-static stiffness is considered, the fundamental natural frequency of the CDPR is around $1.67 \mathrm{~Hz}$.

As the dynamic modulus of elasticity is higher than the quasi-static one, a difference in terms of the oscillation frequency appears in the cable responses. The increase of the oscillation frequency comes from the dynamic stiffening phenomenon, which leads to a reduction in the cable elongation magnitude along the trajectory. It is noteworthy that the dynamic modulus of elasticity tends to increase the natural frequencies.

The CAROCA prototype is also analyzed to check whether the dynamic stiffness has an effect onto the natural frequencies of a smaller CDPR.

The coefficients of the considered cable trapezoidal-velocity profile are $a_{1}=a_{2}=a_{3}=0.2 \mathrm{~m} / \mathrm{s}^{2}$ and $V_{\max }=0.5 \mathrm{~m} / \mathrm{s}$. The end-effector of the CDPR moves from point $P_{1}$ of Cartesian coordinate vector $\mathbf{p}_{1}=[1,0,0.1]^{T} \mathrm{~m}$ to point $P_{3}$ of Cartesian coordinate vector $\mathbf{p}_{3}=[0.74,-0.4,2.6]^{T} \mathrm{~m}$ within $4 \mathrm{~s}$.

From Fig. 16, a resulting forced elongation is observed at a frequency between 10 and $11 \mathrm{~Hz}$, which corresponds to the frequency of the fundamental rigid-body mode of the end-effector suspended onto the global cable stiffness. Based on the experimental data given in Table 1, the corresponding value of the dynamic modulus of elasticity is $E^{\prime}=130.1 \mathrm{GPa} \pm 3.0 \mathrm{GPa}$ at $10 \mathrm{~Hz}$ instead of $E=102 \mathrm{GPa} \pm 2.2 \mathrm{GPa}$ in quasi-static.

The end-effector trajectory can differ up to $1.26 \mathrm{~mm}$ along the $z$-axis, $0.1 \mathrm{~mm}$ along the $x$-axis and $0.05 \mathrm{~mm}$ along the $y$-axis depending on the chosen stiffness. This represents a maximum relative difference of $16.2 \%$ in terms of end-effector tracking errors. The frequency dependency of the dynamic stiffness has the same effects onto the CAROCA as the previous example. Therefore, one can claim that the dynamic and oscillatory responses of the end-effector and cables depend strongly on the dynamic stiffness no matter the size of the CDPR. 


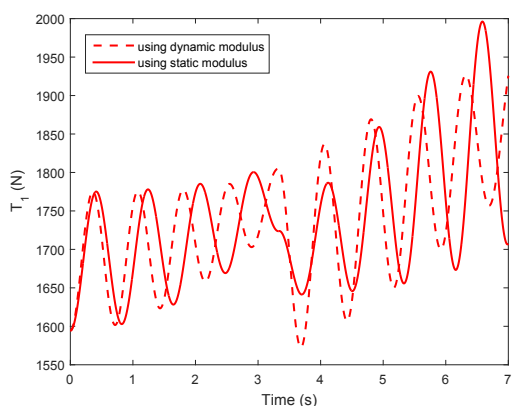

(a) Time history of tension $T_{1}$

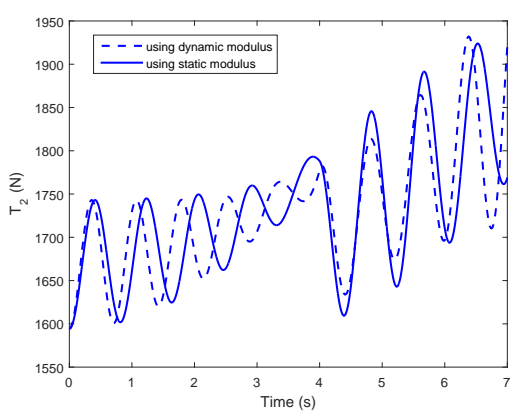

(d) Time history of tension $T_{2}$

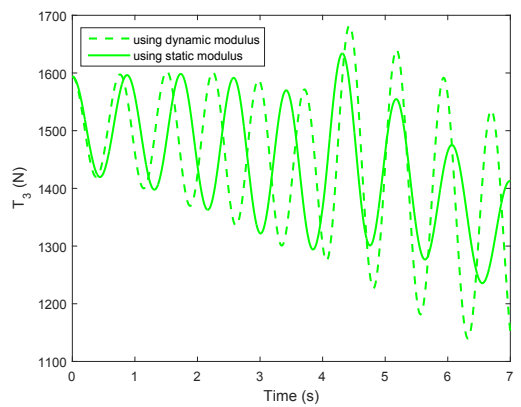

(g) Time history of tension $T_{3}$

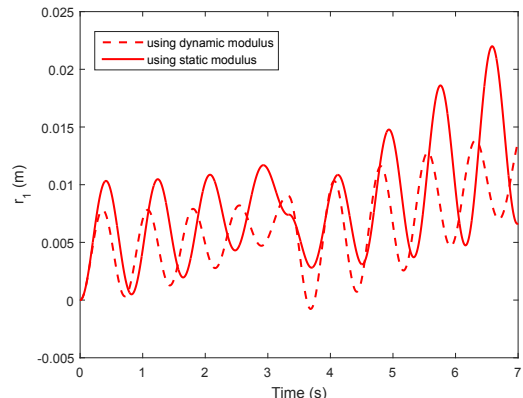

(b) Time history of elongation $r_{1}$

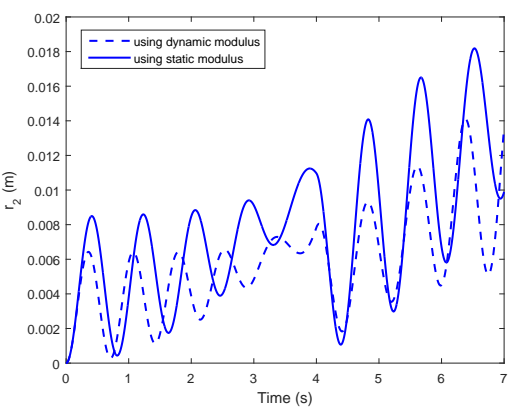

(e) Time history of elongation $r_{2}$

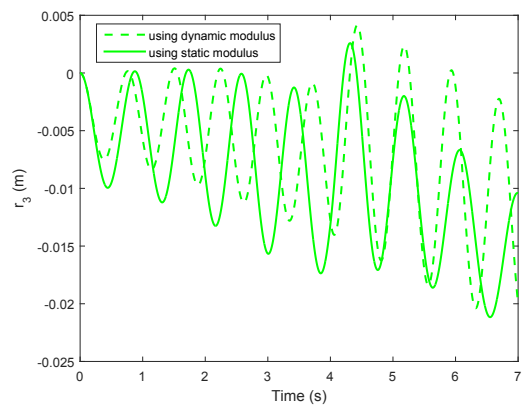

(h) Time history of elongation $r_{3}$

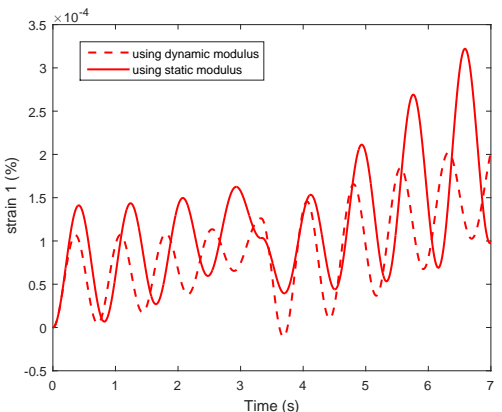

(c) Time history of strain of cable 1

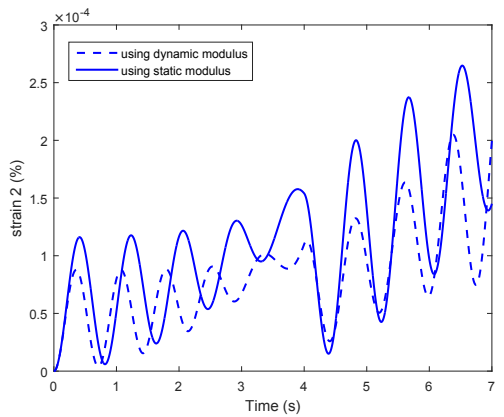

(f) Time history of strain of cable 2

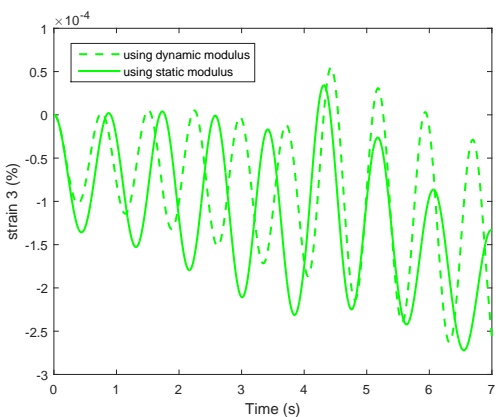

(i) Time history of strain of cable 3

Fig. 13: Comparison between non-linear constitutive formulations using static or dynamic modulus: Tension, elongation and strain

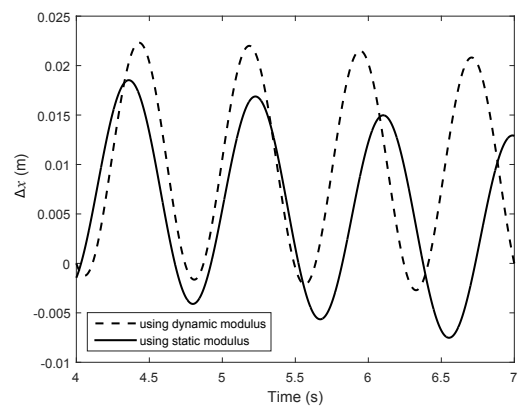

(a) Time history of positioning error along $\mathrm{x}$-axis

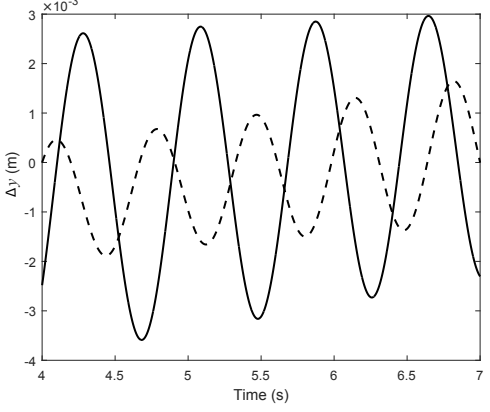

(b) Time history of positioning error along y-axis

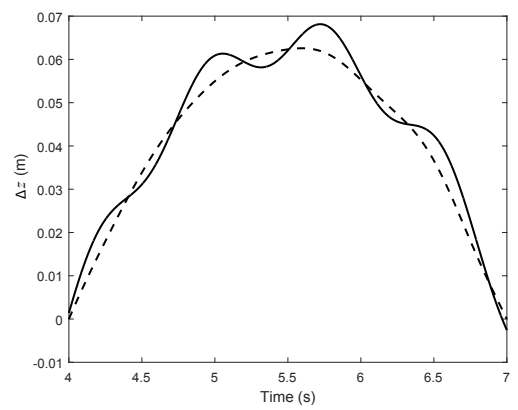

(c) Time history of positioning error along z-axis

Fig. 14: Comparison between non-linear constitutive formulations using static or dynamic modulus: Positioning error of the end-effector along $x$-axis, $y$-axis and $z$-axis 


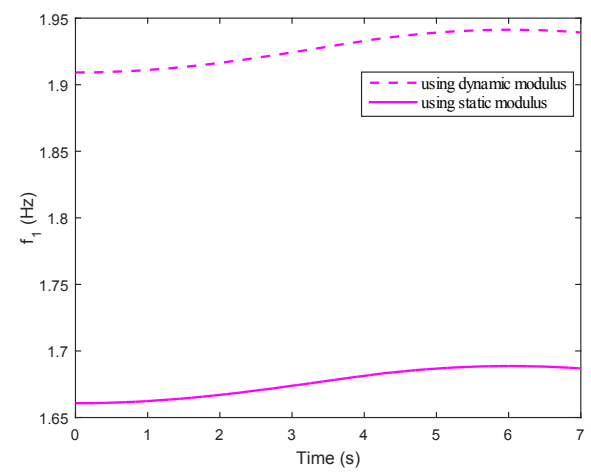

(a) Time history of $f_{1}$

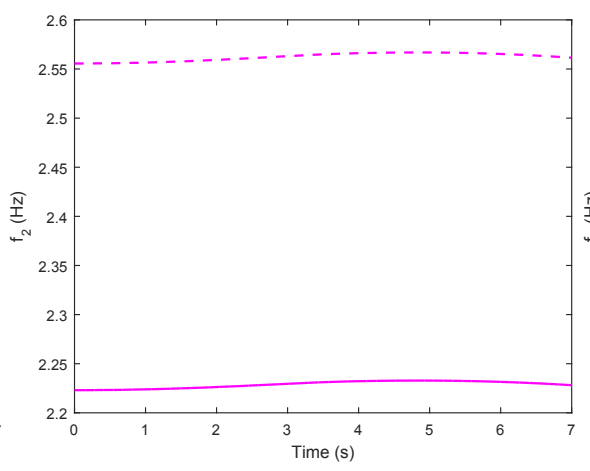

(b) Time history of $f_{2}$

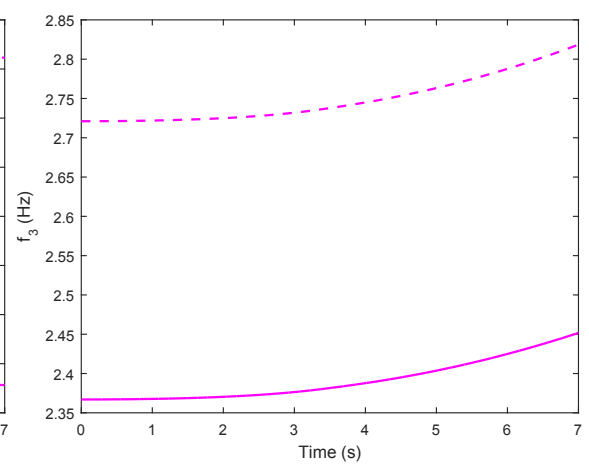

(c) Time history of $f_{3}$

Fig. 15: Comparison between non-linear constitutive formulations using static or dynamic modulus ( $2 \mathrm{~Hz})$ : Natural frequencies

\subsection{Axial cable damping}

To show the influence of axial cable damping on the CDPR dynamic behavior, the results of the simulations with the non-linear tension formulation without (continuous line) and with (pointed line) damping are plotted in Figures 17 and 18. As the oscillation frequency of the CDPR under study is between 1 and $2 \mathrm{~Hz}$, the corresponding dynamic parameters are selected in Table 1 (Section 3). At $2 \mathrm{~Hz}$, the cables damping coefficient is $c=2.08 \times 10^{9} \mathrm{~N} \cdot \mathrm{m}^{-1}$.s and its correspondent dynamic modulus of elasticity is $E^{\prime}=134.8 \mathrm{GPa}$. Using these dynamic parameters, the time histories of cable tension and elongation are presented in Fig. 17. These curves show that the cable response is obviously highly sensitive to cable damping. The oscillations of the damped model decay during the transient phases between 0 to $3.4 \mathrm{~s}$ and between 3.4 to $8 \mathrm{~s}$ whereas the conservative model exhibits no energy loss as expected. The influence of the damping is also significant on the evaluation of the end-effector position tracking error (Fig. 18).

The contribution of these simulations is to show how the cable modulus of elasticity substantially affects the dynamic behavior of CDPR. The results stress the importance of choosing the appropriate method of identification in relation to the correlation between theoretical and experimental results.

\section{Conclusions}

A new non-linear cable tension expression for Cable-Driven Parallel Robots (CDPRs) was proposed in this paper to deal with axial cable vibrations. This non-linear cable tension formulation expresses the relationship between cable tension and the end-effector and its position errors depend on the cable tension model. As the new tension model depends strongly on cable characteristics, Dynamic Mechanical Analysis (DMA) was used to identify carefully the dynamic elastic and damping moduli of some cables. Then, those cable dynamic parameters were integrated into the non-linear cable tension formulation, which allowed us to study the dynamic behavior of a 3 degree-of-freedom CDPR while considering both linear and non-linear cable tension formulations. The comparison between the dynamic responses of the two semi-industrial CDPRs of different sizes shows that substantial differences between the proposed non-linear approach and the classical linear one are more meaningful for large-size CDPRs. Accordingly, the first contribution of this paper deals with a good choice of the cable tension model with regard to the CDPR size and dynamics. Moreover, this paper reveals that the dynamic stiffening of the steel cables has an important effect onto the dynamic and oscillatory motions of the end-effector no matter the CDPR size. This finding supports the idea to adjust each cable model and parameters to the CDPR dynamic model for a good trajectory tracking. Non-redundant CDPRs have been studied in this paper. Future work will generalize this study to redundant six degree-of-freedom CDPRs. The redundancy allows us to increase the minimum cable tension and then to avoid cable slackness along high dynamic trajectories. The modeling approach for such CDPRs requires the integration of tension distribution algorithms that impose to use extended arithmetic in discrete-time control, which may lead to drastic differences in the cable tensions when compared to usual continuous time simulation [35].

\section{References}

[1] Alamdari, A., and Krovi, V., 2016. "Design and analysis of a cable-driven articulated rehabilitation system for gait training”. Journal of Mechanisms and Robotics, 8(5), pp. 051018.1-051018.12.

[2] Homma, K., Fukuda, O., Sugawara, J., Nagata, Y., and Usuba, M., 2003. "A wire-driven leg rehabilitation system: development of a 4-dof experimental system". In 2003 IEEE/ASME International Conference on Advanced Intelligent Mechatronics, 2003. AIM 2003. Proceedings., Vol. 2, IEEE, pp. 908-913. 


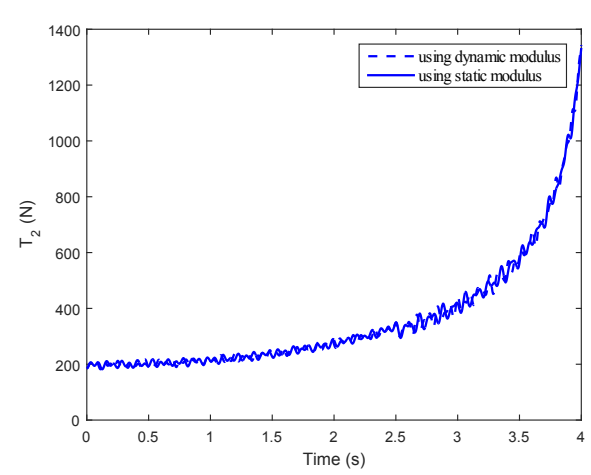

(a) Time history of tension $T_{2}$ : CAROCA

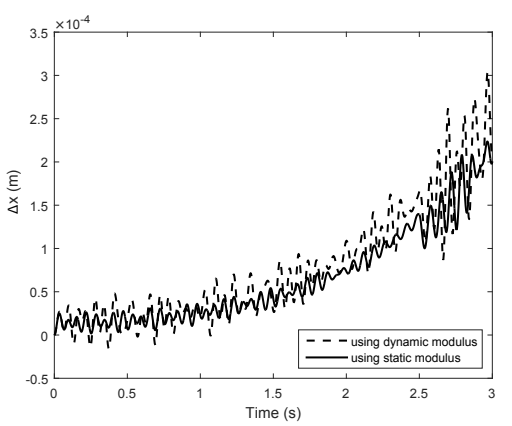

(d) Positioning error along x-axis: CAROCA

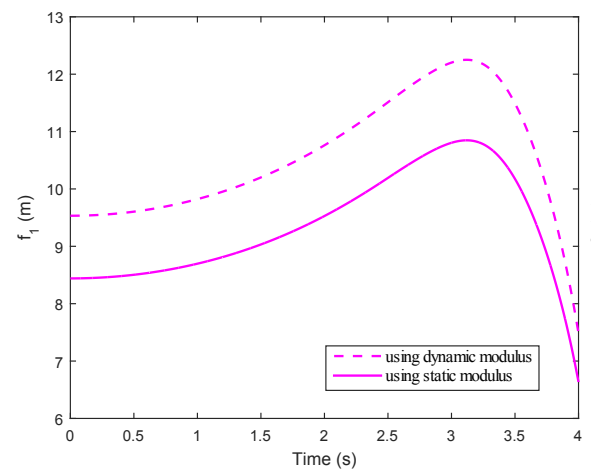

(g) Time history of $f_{1}$ : CAROCA

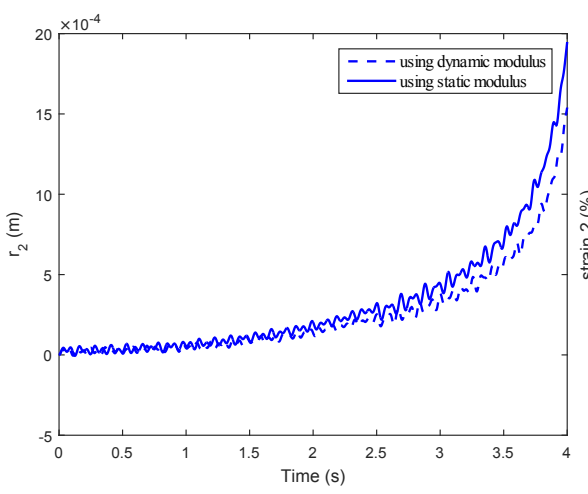

(b) Time history of elongation $r_{2}$ : CAROCA

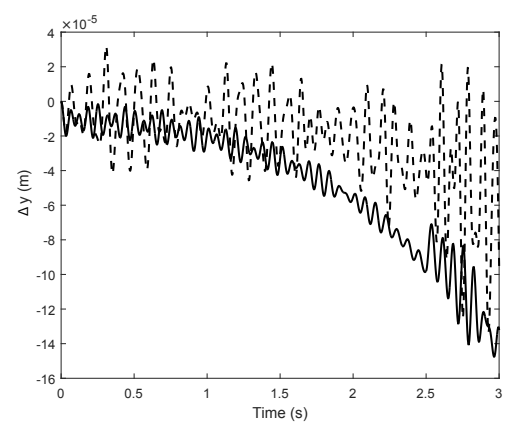

(e) Positioning error along y-axis: CAROCA

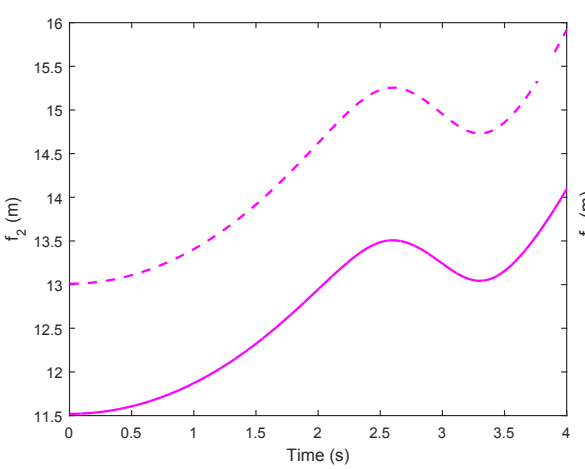

(h) Time history of $f_{2}$ : CAROCA

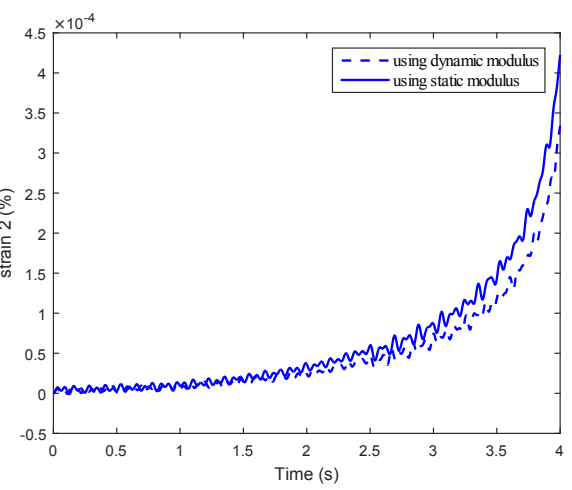

(c) Time history of strain of cable 2: CAROCA

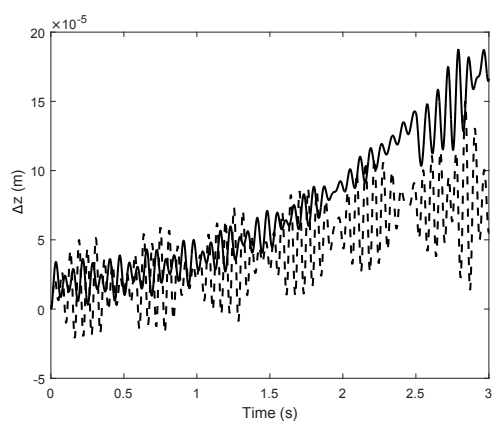

(f) Positioning error along z-axis: CAROCA

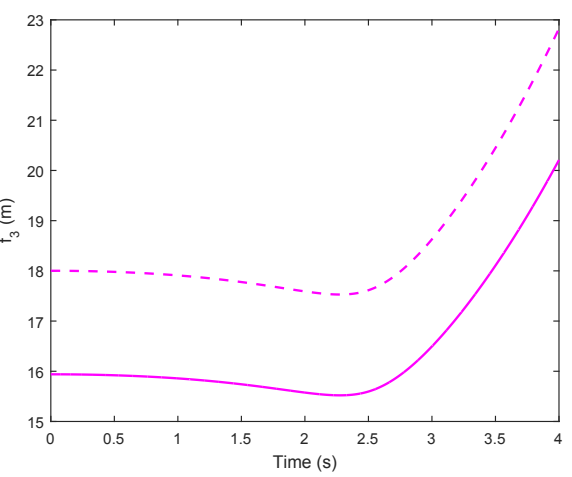

(i) Time history of $f_{3}$ : CAROCA

Fig. 16: Comparison between dynamic responses of the CAROCA while using quasi-static or dynamic modulus (10 Hz)

[3] Merlet, J.-p., and Daney, D., 2010. “A portable, modular parallel wire crane for rescue operations". In 2010 IEEE International Conference on Robotics and Automation (ICRA), IEEE, pp. 2834-2839.

[4] Kawamura, S., Ida, M., Wada, T., and Wu, J.-L., 1995. "Development of a virtual sports machine using a wire drive system-a trial of virtual tennis". In 1995 IEEE/RSJ International Conference on Intelligent Robots and Systems 95.'Human Robot Interaction and Cooperative Robots', Proceedings., Vol. 1, IEEE, pp. 111-116.

[5] Holland, C. S., and Cannon, D. J., 2004. Cable array robot for material handling, Nov. 30. US Patent 6,826,452.

[6] Gagliardini, L., Caro, S., Gouttefarde, M., and Girin, A., 2016. "Discrete reconfiguration planning for cable-driven parallel robots". Mechanism and Machine Theory, 100, pp. 313-337.

[7] Babaghasabha, R., Khosravi, M. A., and Taghirad, H. D., 2015. "Adaptive robust control of fully-constrained cable driven parallel robots". Mechatronics, 25, pp. 27-36.

[8] Diao, X., 2015. "Singularity analysis of fully-constrained cable-driven parallel robots with seven cables". In 2015 IEEE International Conference on Mechatronics and Automation (ICMA), IEEE, pp. 1537-1541.

[9] Abbasnejad, G., and Carricato, M., 2015. "Direct geometrico-static problem of underconstrained cable-driven parallel robots with cables". IEEE Transactions on Robotics, 31(2), pp. 468-478.

[10] Alp, A. B., and Agrawal, S. K., 2002. "Cable suspended robots: design, planning and control". In IEEE International 


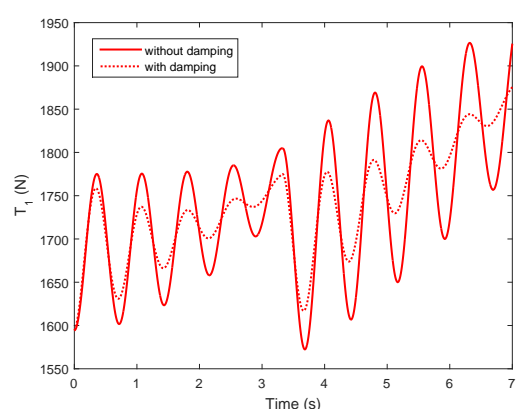

(a) Time history of tension $T_{1}$

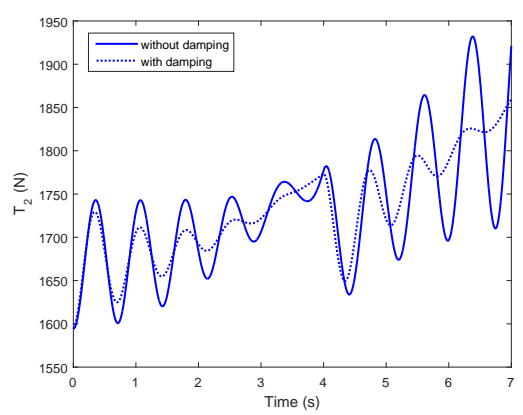

(d) Time history of tension $T_{2}$

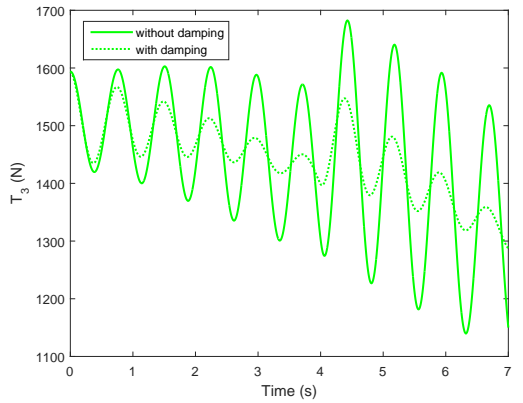

(g) Time history of tension $T_{3}$

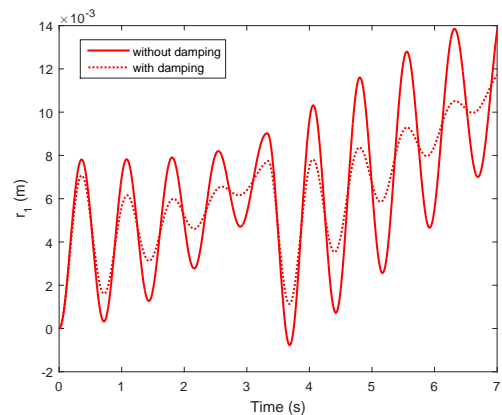

(b) Time history of elongation $r_{1}$

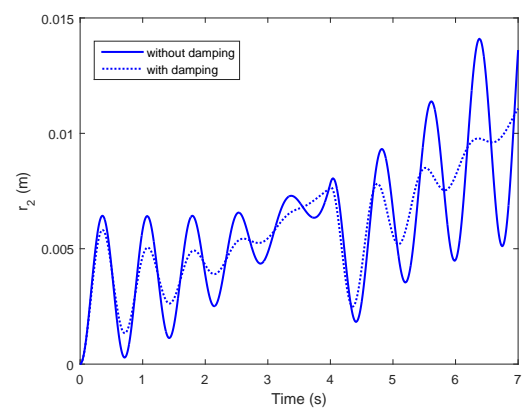

(e) Time history of elongation $r_{2}$

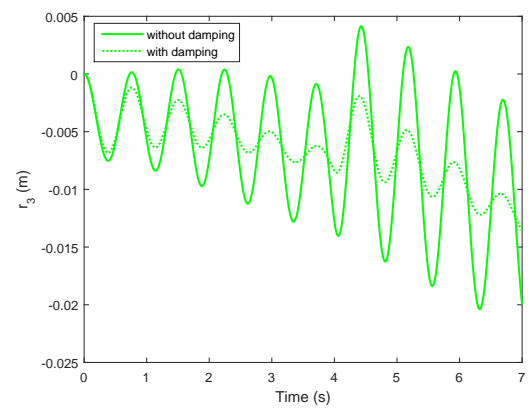

(h) Time history of elongation $r_{3}$

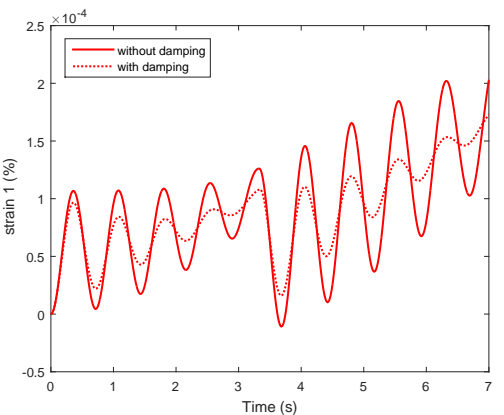

(c) Time history of strain of cable 1

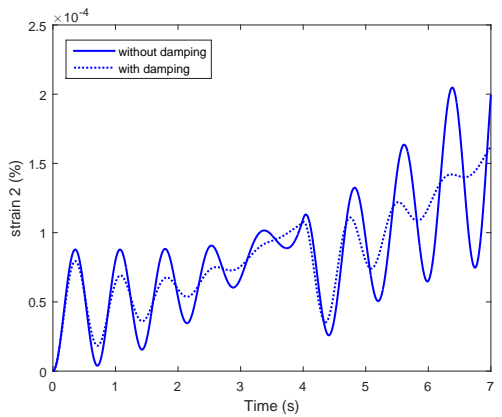

(f) Time history of strain of cable 2

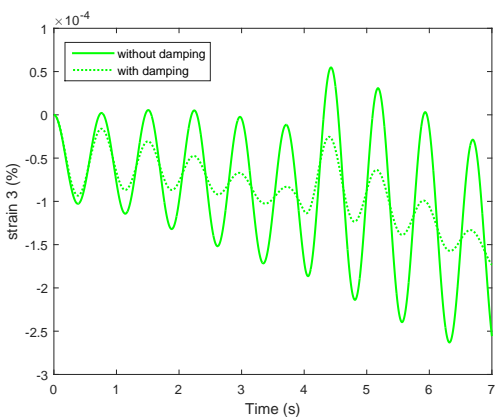

(i) Time history of strain of cable 3

Fig. 17: Comparison between non-linear constitutive formulations with and without damping: Tension, elongation and strain

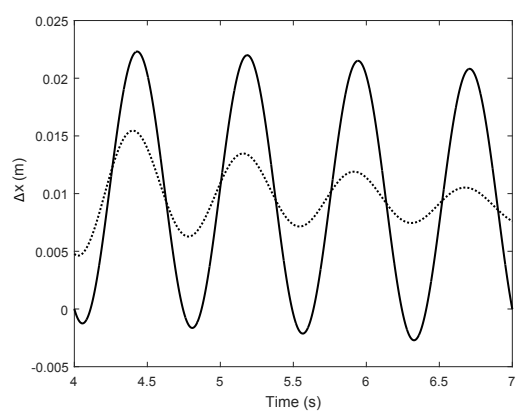

(a) Time history of positioning error along $\mathrm{x}$-axis

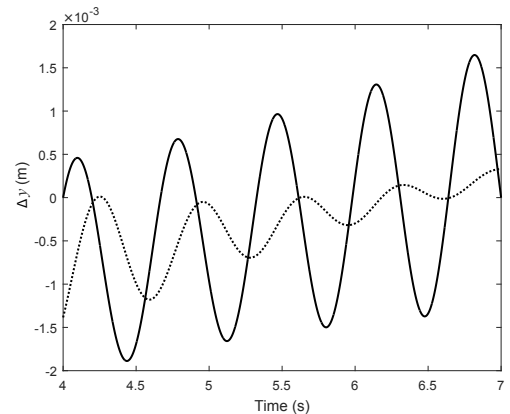

(b) Time history of positioning error along y-axis

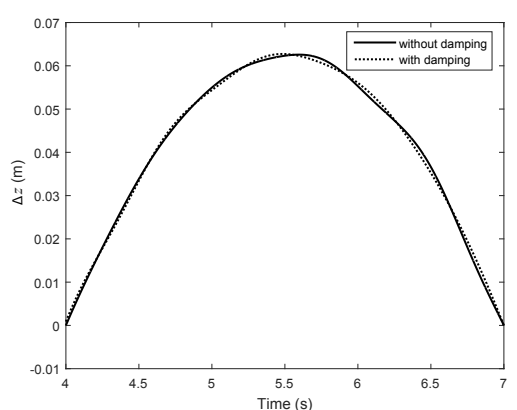

(c) Time history of positioning error along z-axis

Fig. 18: Comparison between non-linear constitutive formulations with and without damping: Positioning error of the end-effector along $x$-axis, $y$-axis and $z$-axis 
Conference on Robotics and Automation, 2002. Proceedings. ICRA’02., Vol. 4, IEEE, pp. 4275-4280.

[11] Carricato, M., and Merlet, J.-P., 2010. "Geometrico-static analysis of under-constrained cable-driven parallel robots". In Advances in Robot Kinematics: Motion in Man and Machine. Springer, pp. 309-319.

[12] Carricato, M., and Merlet, J.-P., 2013. "Stability analysis of underconstrained cable-driven parallel robots". IEEE Transactions on Robotics, 29(1), pp. 288-296.

[13] Kozak, K., Zhou, Q., and Wang, J., 2006. "Static analysis of cable-driven manipulators with non-negligible cable mass". IEEE Transactions on Robotics, 22(3), pp. 425-433.

[14] Arsenault, M., 2013. "Workspace and stiffness analysis of a three-degree-of-freedom spatial cable-suspended parallel mechanism while considering cable mass". Mechanism and Machine Theory, 66, pp. 1-13.

[15] Irvine, H. M., 1992. Cable structures. Dover Publications.

[16] Yuan, H., Courteille, E., and Deblaise, D., 2015. "Static and dynamic stiffness analyses of cable-driven parallel robots with non-negligible cable mass and elasticity". Mechanism and Machine Theory, 85, pp. 64-81.

[17] Diao, X., and Ma, O., 2009. "Vibration analysis of cable-driven parallel manipulators". Multibody system dynamics, 21(4), pp. 347-360.

[18] Yuan, H., Courteille, E., and Deblaise, D., 2014. "Elastodynamic analysis of cable-driven parallel manipulators considering dynamic stiffness of sagging cables". In 2014 IEEE International Conference on Robotics and Automation (ICRA), IEEE, pp. 4055-4060.

[19] Khosravi, M. A., and Taghirad, H. D., 2015. "Dynamic analysis and control of fully-constrained cable robots with elastic cables: variable stiffness formulation”. In Cable-Driven Parallel Robots. Springer, pp. 161-177.

[20] Barrette, G., and Gosselin, C. M., 2005. "Determination of the dynamic workspace of cable-driven planar parallel mechanisms". Journal of Mechanical Design, 127(2), pp. 242-248.

[21] Du, J., and Agrawal, S. K., 2015. "Dynamic modeling of cable-driven parallel manipulators with distributed mass flexible cables". Journal of Vibration and Acoustics, 137(2), pp. 021020.1-021020.8.

[22] Babaz, M., Jezequel, L., Lamarque, C.-H., and Perrard, P., 2016. "Unusual expression of tension of a massless cable with application to the oscillations of a mass suspended to a cable with a variable length". Journal of Sound and Vibration, 363, pp. 446-459.

[23] Gagliardini, L., Caro, S., Gouttefarde, M., and Girin, A., 2015. "A reconfiguration strategy for reconfigurable cable-driven parallel robots". In 2015 IEEE International Conference on Robotics and Automation (ICRA), IEEE, pp. 1613-1620.

[24] Hui, L., 2015. "A giant sagging-cable-driven parallel robot of fast telescope: its tension-feasible workspace of orientation and orientation planning". In Proceedings of the 14th IFToMM World Congress, , pp. 373-381.

[25] Liu, Z., Tang, X., Shao, Z., Wang, L., and Tang, L., 2013. "Research on longitudinal vibration characteristic of the six-cable-driven parallel manipulator in fast". Advances in Mechanical Engineering.

[26] Starossek, U., 1991. "Dynamic stiffness matrix of sagging cable". Journal of engineering mechanics, 117(12), pp. 2815-2828.

[27] Zi, B., Duan, B., Du, J., and Bao, H., 2008. "Dynamic modeling and active control of a cable-suspended parallel robot". Mechatronics, 18(1), pp. 1-12.

[28] Bedoustani, Y. B., Taghirad, H. D., and Aref, M. M., 2008. "Dynamics analysis of a redundant parallel manipulator driven by elastic cables". In 10th International Conference on Control, Automation, Robotics and Vision, 2008. ICARCV 2008., IEEE, pp. 536-542.

[29] Miyasaka, M., Haghighipanah, M., Li, Y., and Hannaford, B., 2016. "Hysteresis model of longitudinally loaded cable for cable driven robots and identification of the parameters". In 2016 IEEE International Conference on Robotics and Automation (ICRA), IEEE, pp. 4051-4057.

[30] Miermeister, P., Kraus, W., Lan, T., and Pott, A., 2015. "An elastic cable model for cable-driven parallel robots including hysteresis effects". In Cable-Driven Parallel Robots. Springer, pp. 17-28.

[31] Menard, K. P., 2008. Dynamic mechanical analysis: a practical introduction. CRC press.

[32] Haddad, Y. M., 1995. "Viscoelasticity of engineering materials". Chapman \& Hall, 2-6 Boundary Row, London, SE 18 $H N, U K, 1995.378$.

[33] Carfagni, M., Lenzi, E., and Pierini, M., 1998. “The loss factor as a measure of mechanical damping”. In Proceedings-spie the international society for optical engineering, Vol. 1, Spie International Society for Optical, pp. 580-284.

[34] Weber, X., Cuvillon, L., and Gangloff, J., 2014. "Active vibration canceling of a cable-driven parallel robot using reaction wheels". In 2014 IEEE/RSJ International Conference on Intelligent Robots and Systems, IEEE, pp. 1724-1729.

[35] Merlet, J.-P., 2017. "Simulation of discrete-time controlled cable-driven parallel robots on a trajectory". IEEE Transactions on Robotics. 